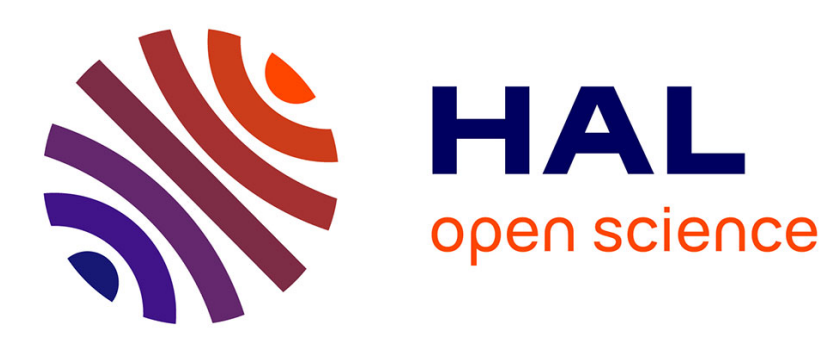

\title{
Interprétation quantique des diverses résonances observées lors de la diffusion de photons optiques et de radiofréquence par un atome

\author{
C. Cohen-Tannoudji, S. Haroche
}

\section{- To cite this version:}

C. Cohen-Tannoudji, S. Haroche. Interprétation quantique des diverses résonances observées lors de la diffusion de photons optiques et de radiofréquence par un atome. Journal de Physique, 1969, 30 (1), pp.125-144. 10.1051/jphys:01969003001012500 . jpa-00206756

\section{HAL Id: jpa-00206756 https://hal.science/jpa-00206756}

Submitted on 1 Jan 1969

HAL is a multi-disciplinary open access archive for the deposit and dissemination of scientific research documents, whether they are published or not. The documents may come from teaching and research institutions in France or abroad, or from public or private research centers.
L'archive ouverte pluridisciplinaire HAL, est destinée au dépôt et à la diffusion de documents scientifiques de niveau recherche, publiés ou non, émanant des établissements d'enseignement et de recherche français ou étrangers, des laboratoires publics ou privés. 


\title{
INTERPRÉTATION QUANTIQUE DES DIVERSES RÉSONANGES OBSERVÉES LORS DE LA DIFFUSION DE PHOTONS OPTIQUES ET DE RADIOFRÉQUENGE PAR UN ATOME
}

\author{
par G. COHEN-TANNOUDJI et S. HAROCHE, \\ Faculté des Sciences de Paris, Laboratoire de Spectroscopie Hertzienne de l'E.N.S., \\ associé au C.N.R.S., 24, rue Lhomond, Paris, $5^{\mathrm{e}}$. \\ (Reçu le 18 octobre 1968.)
}

\begin{abstract}
Résumé. - On présente une théorie quantique de divers types de résonances apparaissant sur la lumière diffusée par un atome interagissant avec un champ de radiofréquence (cas des expériences de pompage optique longitudinal et transversal). On associe à tout processus de diffusion de photons optiques et de radiofréquence un diagramme de Feynman comportant éventuellement un ou plusieurs états intermédiaires résonnants. On montre que certaines résonances sont dues à l'interférence entre deux diagrammes. Une resommation des diagrammes aux différents ordres permet de rendre compte des élargissements et déplacements radiatifs des résonances.
\end{abstract}

\begin{abstract}
A quantum theory of the various types of resonances appearing on the light scattered by an atom interacting with a radiofrequency field (longitudinal and transverse optical pumping experiments) is given. Each scattering process of optical and RF photons is associated a Feynman diagram containing eventually one or several intermediate resonant states. Some resonances are shown to be due to the interference between two diagrams. By a resummation of the diagrams at different orders, it is possible to explain the radiative shifts and broadenings of the resonances.
\end{abstract}

Introduction. - Dans les expériences de double résonance, les atomes étudiés sont soumis à l'action simultanée d'un faisceau lumineux (permettant d'orienter et de détecter le système atomique) et d'un champ de radiofréquence (induisant des transitions entre les sous-niveaux Zeeman). Sur le plan théorique, le champ optique a souvent fait l'objet d'un traitement quantique [1], [2], mais, à part quelques exceptions $\left(^{1}\right)$, le champ de radiofréquence a toujours été traité classiquement.

L'aspect corpusculaire du champ de radiofréquence se manifeste cependant avec éclat dans les transitions à plusieurs quanta [4]. En appliquant le principe de conservation de l'énergie et du moment angulaire au processus d'absorption de $n$ quanta de radiofréquence

(1) Voir, par exemple, R. Wallace [3]. Cet auteur ne s'intéresse cependant qu'à l'un des deux types de résonances étudiées dans cet article (transitions réelles à plusieurs quanta). N'effectuant pas de resommation des diagrammes, il n'obtient pas par ailleurs les effets de saturation (déplacement et élargissement des résonances). par un atome, on déduit très aisément la position des résonances correspondantes. Récemment, de nouveaux types de résonance magnétique ont été observés en pompage optique transversal [5], [6], [7], [8], [9]. Les caractéristiques de ces résonances les différencient nettement des précédentes et leur interprétation en termes de photons de radiofréquence soulève des problèmes difficiles.

Le but de cet article est de présenter une théorie entièrement quantique de tous ces phénomènes : elle traite de façon symétrique les photons optiques et les photons de radiofréquence et permet d'interpréter simultanément les deux types de résonance mentionnés plus haut (position, intensités, élargissements et déplacements radiatifs des résonances, modulations apparaissant sur les signaux optiques).

Dans une première partie, on associe un diagramme de Feynman [10] à tout processus de diffusion de photons optiques ou de radiofréquence par un atome. Pour passer de l'état initial à l'état final de la diffusion, le système effectue des transitions vers des états inter- 
médiaires de diffusion qui sont soit résonnants (transitions réelles), soit non résonnants (transitions virtuelles). Les transitions à plusieurs quanta de type Winter [4] peuvent être décrites par un diagramme de Feynman unique comportant deux états intermédiaires résonnants différents et décrivant l'absorption réelle d'un photon optique suivie de l'absorption réelle d'un ou plusieurs quanta de radiofréquence. Pour interpréter les nouvelles résonances, il est nécessaire de faire appel à l'interférence entre deux diagrammes de Feynman distincts correspondant aux mêmes états initial et final de diffusion et comportant chacun un seul état intermédiaire résonnant, différent pour chacun des diagrammes, les processus physiques associés correspondant à une absorption réelle d'un photon optique et d'un ou plusieurs photons de radiofréquence par l'atome ${ }^{(2}$ ).

Dans une deuxième partie, on effectue une resommation complète des diagrammes correspondant aux mêmes états initial et final de diffusion et on montre que l'effet de cette resommation revient principalement à renormaliser les propagateurs des états intermédiaires résonnants de la diffusion. Il est possible alors d'effectuer un calcul complet des signaux de double résonance et notamment de rendre compte des effets d'élargissements et de déplacements radiatifs.

$\mathrm{Au}$ cours de cette resommation, nous sommes amenés tout naturellement à considérer que les photons optiques sont diffusés par le système global « atome + champ de radiofréquence ». Ce concept d'atome " habillé » par des photons de radiofréquence se révèle très fructueux, en particulier lorsque le couplage avec la radiofréquence est trop grand pour être traité comme une perturbation. Nous développerons cette idée dans un second article et montrerons en particulier que les propriétés physiques de l'atome " habillé » (moment magnétique, spectre d'absorption) sont nettement différentes de celles de l'atome «nu ».

I. Position du problème. Etude qualitative. A. Notations. - 1. Le système atomique. - Il comporte un état fondamental $|f\rangle$ et un état excité $|e\rangle$, d'énergie $E_{0}$, de spin $J$, décomposé par un champ $H_{0}$ parallèle à $\mathrm{Oz}$ en sous-niveaux Zeeman $\left|e_{m}\right\rangle$ distants de $\omega_{0}=-\gamma H_{0} \quad(\gamma$, rapport gyromagnétique).

L'état $|f\rangle$ n'est pas couplé à $H_{0}$, soit parce qu'il est diamagnétique, soit parce qu'il a une structure purement nucléaire, négligeable devant $\omega_{0}(\hbar=1)\left({ }^{3}\right)$. L'hamiltonien atomique s'écrit donc :

$$
\mathscr{H}_{\mathrm{at}}=\left(E_{0}+\omega_{0} J_{z}\right) P e
$$

$P e$ représentant le projecteur dans l'état excité.

$\left(^{2}\right)$ Khodovoy [11] a également envisagé des processus faisant intervenir l'absorption simultanée de photons optiques et de radiofréquence. Mais il ne met pas l'accent sur l'interférence entre amplitudes de transition et ne procède pas lui non plus à une resommation de diagrammes.

$\left(^{3}\right)$ Si l'état fondamental est diamagnétique, le caractère dipolaire de la transition optique fait que $J$ est
2. Le champ de radiofréquence. - Nous supposerons qu'il oscille sur un mode unique de pulsation $\omega$ et de polarisation $\boldsymbol{\epsilon}$, les opérateurs de création et d'annihilation d'un photon dans ce mode étant $a^{\dagger}$ et $a$. L'hamiltonien du champ s'écrit :

$$
\mathscr{H}_{\mathrm{RF}}=\omega a^{\dagger} a \text {. }
$$

Les états propres de $\mathscr{H}_{0}=\mathscr{H}_{\mathrm{at}}+\mathscr{H}_{\mathrm{RF}}$ sont les états $|f, n\rangle$ (d'énergie $n \omega$ ) et $\left|e_{m}, n\right\rangle$ d'énergie $E_{0}+m \omega_{0}+n \omega$ (atome dans l'état fondamental ou excité en présence de $n$ photons).

La figure 1 représente les niveaux $\left|e_{ \pm}, n\right\rangle$ en fonction de $H_{0}(J=1 / 2)$. Il apparaît une infinité de

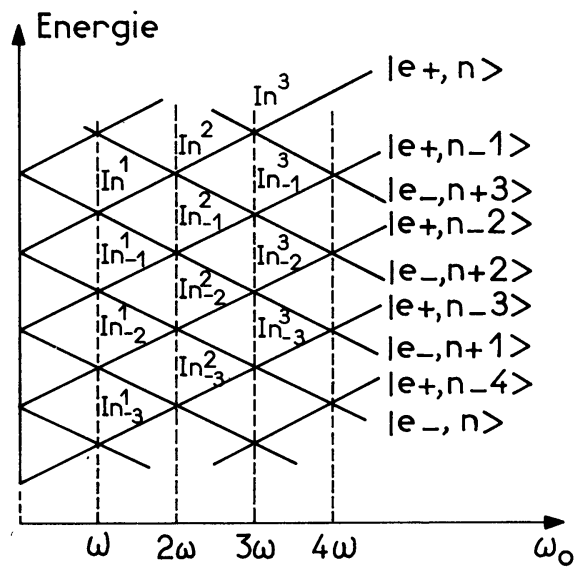

FIG. 1.

croisements de niveaux $I_{n}^{p}$ alignés verticalement pour toutes les valeurs $\omega_{0}=p \omega$.

Le couplage $V$ entre l'atome et la radiofréquence n'est appréciable que dans l'état excité et est donné par (cf. appendice 1) :

$$
V=\lambda \mathbf{J}\left(a \boldsymbol{\epsilon}+a^{\dagger} \boldsymbol{\epsilon}^{*}\right) P e
$$

la constante de couplage $\lambda$ est donnée dans le cas d'une polarisation $\boldsymbol{\epsilon}$ linéaire par la relation :

$$
\lambda=\gamma H_{1} / 2 \sqrt{N}
$$

$H_{1}$ et $N$ étant l'amplitude moyenne et le nombre moyen de photons du champ.

La structure de $V$ est très simple et correspond soit à l'absorption (terme $a$ ), soit à l'émission (terme $a^{\dagger}$ ) d'un photon de radiofréquence, les transitions correspondantes conservant le moment cinétique global de l'atome et du champ (cf. appendice 1).

3. Le champ optique. - A tout mode optique de vecteur d'onde $\mathbf{k}$ et de polarisation $\mathbf{e}_{\lambda}$, nous associons

nécessairement égal à 1 . Dans la suite, nous envisagerons souvent, pour simplifier, le cas où $J=1 / 2$. L'état fondamental est alors nécessairement paramagnétique et $|f\rangle$ représente l'un des sous-niveaux de cet état. 
un photon $|\mathbf{k} \boldsymbol{\lambda}\rangle$ d'énergie $E_{k}$, état propre de l'hamiltonien optique :

$$
\mathscr{H}_{\mathrm{opt}}=\sum_{\mathbf{k}, \lambda} E_{k} a_{\mathbf{k} \lambda}^{\dagger} a_{\mathbf{k} \lambda} .
$$

L'interaction dipolaire électrique $\mathscr{H}_{\mathrm{I}}$ entre le système atomique et le champ optique n'agit pas sur les variables de radiofréquence et ne couple entre eux que les états $|f n \mathbf{k} \lambda\rangle$ et $\left|e_{m}, n\right\rangle$ correspondant au même nombre $n$ :

$$
\left\langle f n \mathbf{k} \lambda\left|\mathscr{H}_{\mathbf{I}}\right| e_{m}, n^{\prime}\right\rangle=\delta_{n, n^{\prime}} B_{m}
$$

avec :

$$
\left\langle f \mathbf{k} \lambda\left|\mathscr{H}_{\mathbf{I}}\right| e_{m}\right\rangle=B_{m} .
$$

B. Diagrammes de Feynman [10]. - Tout processus de diffusion d'un photon optique ou de radiofréquence par l'atome peut être représenté par un diagramme de Feynman, se lisant de bas en haut, et où $|f\rangle$ est représenté par un trait pointillé, $\left|e_{m}\right\rangle$ par un trait plein, les photons optiques par des lignes brisées, les photons de radiofréquence par des lignes ondulées, l'interaction dipolaire électrique $\mathscr{H}_{\mathrm{I}}$ par un vertex carré et l'interaction magnétique $V$ par un vertex rond.

$\left(D_{1}\right)$ représente la diffusion d'un photon optique

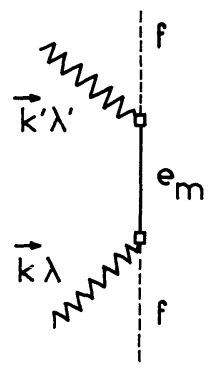

DIAGRAMME: $\mathrm{D}_{\mathbf{1}}$.

$\left(\mathbf{k} \boldsymbol{\lambda} \rightarrow \mathbf{k}^{\prime} \lambda^{\prime}\right)$ avec excitation intermédiaire de l'atome dans l'état $e_{m}$.

L'énergie $E_{k}$ de l'état initial doit être égale à celle, $E_{k^{\prime}}$, de l'état final de la diffusion. Par contre, l'énergie de l'état intermédiaire $E_{0}+m \omega_{0}$ peut être différente de $E_{k}=E_{k^{\prime}}$ pourvu que le temps de séjour $\tau$ dans cet état soit suffisamment court :

$$
\left(\tau \lesssim\left|\frac{1}{E_{k}-E_{0}-m \omega_{0}}\right|\right) \text {. }
$$

Suivant que $E_{0}+m \omega_{0}$ est égal ou non à $E_{k}$ (à la largeur naturelle $\Gamma$ du niveau excité près), nous dirons que la transition intermédiaire est réelle ou virtuelle. L'amplitude de diffusion associée à $\left(D_{1}\right)$ s'obtient en sommant sur toutes les durées possibles de la transition intermédiaire. Il en résulte (cf. II.A.9) un dénominateur d'énergie en $\frac{1}{E_{k}-E_{0}-m \omega_{0}+i \frac{\Gamma}{2}}$, ce qui montre que l'amplitude de diffusion passe par une valeur résonnante lorsque la transition intermédiaire est réelle.

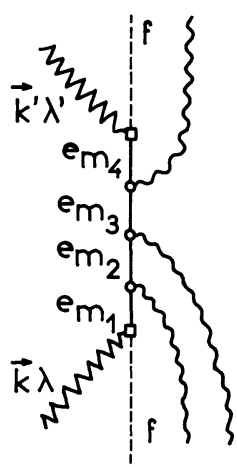

DIAGRAMme $\mathrm{D}_{2}$.

$\left(\mathrm{D}_{2}\right)$ possède quatre états intermédiaires et représente la diffusion d'un photon $|\mathbf{k} \lambda\rangle$ par un atome qui absorbe deux, puis réémet un photon de radiofréquence dans l'état excité ( $\left.{ }^{4}\right)$, la conservation de l'énergie à l'issue de la diffusion s'écrivant dans ce cas :

$$
E_{k^{\prime}}+\omega=E_{k}+2 \omega \text {. }
$$

Supposons maintenant $J=1 / 2$; les énergies des états intermédiaires de la diffusion sont données en fonction du champ magnétique par la figure 1 : pour un champ $H_{0}$ donné, il ne peut y avoir de transition intermédiaire réelle que si l'énergie $E_{k}+n \omega$ de l'état initial $\mid f n \mathbf{k} \lambda>$ coïncide avec l'une des valeurs propres de $\mathscr{H}_{0}$ dans ce champ. Il en résulte qu'en général un état intermédiaire seulement pourra être résonnant, sauf si $\omega_{0}=p \omega$, auquel cas il peut y avoir transition réelle vers deux niveaux distincts de même énergie qui se croisent $(5)$; ceci conduit à l'existence de processus résonnants que nous nous proposons d'étudier maintenant à l'ordre le plus bas où ils se manifestent.

C. Transitions Réelles a un ou Plusieurs QuANTA DE RADIOFRÉQUENGE [4]. - Envisageons tout d'abord des photons optiques incidents et diffusés ayant un état de polarisation circulaire respectivement $\sigma^{-}$et $\sigma^{+}$ (avec les notations de la référence [2], il s'agit de polarisations non cohérentes, chacune d'entre elles ne

(4) Rappelons que nous n'envisageons que les photons de radiofréquence appartenant à un mode donné ; nous négligeons les processus d'interaction avec les photons des autres modes : dans $\mathrm{D}_{2}$ et dans les diagrammes suivants, nous n'avons représenté que les photons de radiofréquence absorbés ou émis. En toute rigueur, il aurait fallu également représenter le très grand nombre de photons "spectateurs " de mêmes fréquence et polarisation dont la présence stimule l'émission dans ce mode et permet de négliger l'émission spontanée dans les autres modes.

$\left.{ }^{5}\right)$ Il ne peut y avoir de transitions réelles vers deux niveaux dont l'énergie diffère de $\omega$, car nous supposons remplie la condition $\omega \gg \Gamma$. 
permettant d'exciter ou de détecter qu'un seul sousniveau de l'état excité) :

$$
\begin{array}{r}
\left\langle f \mathbf{k} \sigma_{-}\left|\mathscr{H}_{\mathrm{I}}\right| e_{+}\right\rangle=B_{+}=0 \\
\left\langle f \mathbf{k} \sigma_{-}\left|\mathscr{H}_{\mathrm{I}}\right| e_{-}\right\rangle=B_{-} \neq 0 \\
\left\langle f \mathbf{k}^{\prime} \sigma_{+}\left|\mathscr{H}_{1}\right| e_{+}\right\rangle=B_{+}^{\prime} \neq 0 \\
\left\langle f \mathbf{k}^{\prime} \sigma_{+}\left|\mathscr{H}_{\mathrm{I}}\right| e_{-}\right\rangle=B_{-}^{\prime}=0 .
\end{array}
$$

Il en résulte qu'en absence de radiofréquence l'amplitude de diffusion $\mathbf{k} \sigma_{-} \rightarrow \mathbf{k}^{\prime} \sigma_{+}$est nulle.

Les diagrammes $\left(\mathrm{D}_{3}\right)$ et $\left(\mathrm{D}_{4}\right)$ montrent comment l'interaction de l'état excité avec un champ de radio-

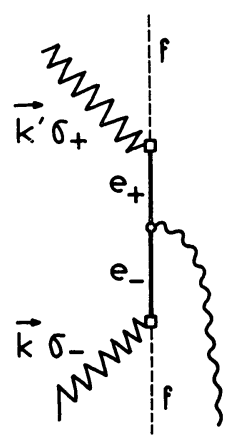

DIAGRAMME $\mathrm{D}_{3}$.

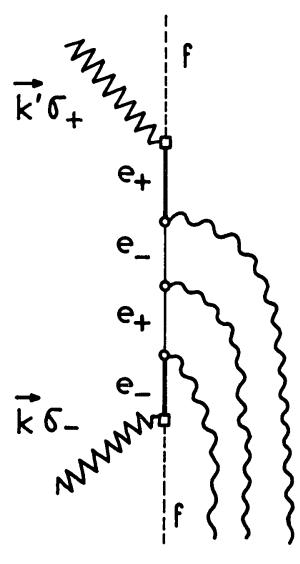

Diagramme $\mathrm{D}_{\mathbf{4}}$. fréquence peut rendre cette amplitude non nulle et lui donner un caractère résonnant pour certaines valeurs de $\omega_{0}$.

Dans $\left(\mathrm{D}_{3}\right)$, l'atome est excité dans $\left|e_{-}, n\right\rangle$ par absorption d'un photon optique $\mathbf{k} \sigma_{-}$; puis il absorbe un photon de radiofréquence et passe dans l'état $\left|e_{+}, n-1\right\rangle$ à partir duquel a lieu l'émission d'un photon optique $\mathbf{k}^{\prime} \sigma_{+}$.

Nous supposerons le champ de radiofréquence tournant circulaire droit par rapport à $H_{0}\left(\sigma^{+}\right)$ou linéaire et perpendiculaire à $H_{0}(\sigma)$. Dans les deux cas, le couplage $V$ entre les deux états intermédiaires $\left|e_{-}, n\right\rangle$ et $\left|e_{+}, n-1\right\rangle$ est possible (cf. appendice 1).

L'amplitude de diffusion correspondant à $\left(\mathrm{D}_{3}\right)$ sera importante, si les deux transitions intermédiaires sont réelles, c'est-à-dire si (condition de double résonance) :

a) $E_{k}=E_{0}-\frac{\omega_{0}}{2}$ (résonance optique);

b) $\omega_{0}=\omega$ (résonance magnétique).

Dans $\left(\mathbf{D}_{4}\right)$, l'atome subit une transition vers l'état $\left|e_{-}, n\right\rangle$, puis trois transitions successives de radiofréquence qui le conduisent à l'état $\left|e_{+}, n-3\right\rangle$ avant de réémettre le photon optique diffusé. Nous avons alors quatre états intermédiaires de diffusion. Si

$E_{k}=E_{0}-\frac{\omega_{0}}{2} \quad(\grave{a} \quad \Gamma$ près $)$ l'énergie est conservée pour le premier état intermédiaire. Si $\omega_{0}=3 \omega$, elle l'est également pour le quatrième état intermédiaire.
Les deuxième et troisième états intermédiaires ont alors une énergie nécessairement différente de l'énergie initiale : nous avons, dans l'état excité, une transition réelle à trois quanta qui s'effectue par l'intermédiaire de deux transitions virtuelles. Il est facile de voir que $\left(\mathrm{D}_{4}\right)$ n'existe pas pour la polarisation $\sigma^{+}$du champ de radiofréquence. En effet, le couplage entre les deuxième et troisième états intermédiaires $\left|e_{+}, n-1\right\rangle$ et $\left|e_{-}, n-2\right\rangle$ est alors nul. Par contre, $\left(\mathrm{D}_{4}\right)$ existe pour la polarisation $\sigma$.

On peut imaginer aisément des diagrammes faisant intervenir plus de trois quanta de radiofréquence et correspondant à des processus de double résonance, c'est-à-dire impliquant :

a) Une « résonance optique » qui se produit lorsque l'énergie est conservée à l'absorption du photon incident;

b) Une « résonance magnétique » qui se produit lorsque l'énergie est conservée à l'issue des transitions de radiofréquence avant l'émission du photon optique diffusé. Les diagrammes de diffusion correspondants comportent deux états intermédiaires simultanément résonnants, condition qui ne peut être remplie qu'en un point de croisement de deux niveaux de $\mathscr{H}_{0}$.

D'autre part, il faut évidemment que ces deux niveaux de $\mathscr{H}_{0}$ qui se croisent soient couplés par $V$ puisqu'ils figurent sur le même diagramme. La conservation du moment cinétique global entraîne que, dans le cas d'un champ de radiofréquence tournant $\sigma_{+}$, ceci ne se produit qu'aux points $I_{n}^{1}$ tels que $\omega_{0}=\omega$; dans le cas d'un champ à polarisation linéaire $\sigma$, il faut que l'on soit aux points $I_{n}^{2 p+1}$ tels que :

$$
\omega_{0}=(2 p+1) \omega
$$

Par contre, pour une telle polarisation, aucun processus résonnant de ce type n'est possible aux points $I_{n}^{2 p}$ tels que $\omega_{0}=2 p \omega$, car les niveaux $\left|e_{-}, n\right\rangle$ et $\left|e_{+}, n-2 p\right\rangle$ qui s'y croisent ne sont alors couplés à aucun ordre (cf. appendice 1).

Nous mettons cependant en évidence, au paragraphe suivant, de nouveaux types de diffusion conduisant à des résonances pour ces valeurs du champ.

D. INTERFÉRENGES ENTRE AMPLITUDES DE TRANSITION : NOUVEAUX PROCESSUS RÉSONNANTS. - Supposons maintenant que les photons optiques incidents (diffusés) ont une polarisation cohérente $\mathbf{e}_{\lambda}\left(\mathbf{e}_{\lambda^{\prime}}\right)$ qui permet d'exciter (de détecter) l'atome dans chacun des deux sous-niveaux Zeeman de l'état excité. Nous avons alors :

$$
\left\{\begin{array}{l}
\left\langle f \mathbf{k} \lambda\left|\mathscr{H}_{\mathrm{I}}\right| e_{ \pm}\right\rangle=B_{ \pm} \neq 0 \\
\left\langle f \mathbf{k}^{\prime} \lambda^{\prime}\left|\mathscr{H}_{\mathrm{I}}\right| e_{ \pm}\right\rangle=B_{ \pm}^{\prime} \neq 0 .
\end{array}\right.
$$

En l'absence de radiofréquence, le processus de diffusion $|\mathbf{k} \boldsymbol{\lambda}\rangle \rightarrow\left|\mathbf{k}^{\prime} \lambda^{\prime}\right\rangle$ peut maintenant s'effectuer par l'intermédiaire de chacun des deux états $\left|e_{+}\right\rangle$ 


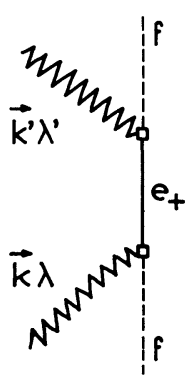

$\alpha)$

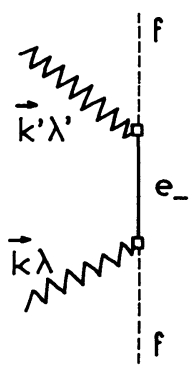

$\beta)$
DIAGRAMME $\mathrm{D}_{5}$.

et $\left|e_{-}\right\rangle$. Aux deux diagrammes $\left(\mathrm{D}_{5} \alpha\right)$ et $\left(\mathrm{D}_{5} \beta\right)$ qui partent du même état initial pour aboutir au même état final sont associées deux amplitudes de diffusion, $A_{+}$et $A_{-}$, qu'il faut ajouter pour obtenir l'amplitude de diffusion totale $A=A_{+}+A_{-}$.

La probabilité de transition $W_{\mathbf{k} \lambda \rightarrow \mathbf{k}^{\prime} \lambda^{\prime}}$ s'écrit alors : $W_{\mathbf{k} \lambda \rightarrow \mathbf{k}^{\prime} \lambda^{\prime}}=|A|^{2}=\left|A_{+}\right|^{2}+\left|A_{-}\right|^{2}+2 \mathscr{R} \mathrm{e} A_{+} A_{-}^{*}$.

$A_{+}$n'est important que si la transition intermédiaire correspondante est réelle, c'est-à-dire si :

$$
\left|E_{k}-\left(E_{0}+\frac{\omega_{0}}{2}\right)\right| \lesssim \Gamma \text {. }
$$

De même, $A_{-}$n'est important que si :

$$
\left|E_{k}-\left(E_{0}-\frac{\omega_{0}}{2}\right)\right| \lesssim \Gamma \text {. }
$$

Le terme d'interférence de $W_{\mathbf{k} \lambda \rightarrow \mathbf{k}^{\prime} \lambda^{\prime}}$ est maximum lorsque $A_{+}$et $A_{-}$sont simultanément résonnants, c'est-à-dire si $\omega_{0} \lesssim \Gamma$, c'est-à-dire encore au voisinage du croisement des deux niveaux en champ nul (effet Hanle) [12].

Dès que $\omega_{0}>\Gamma$, les effets d'interférence précédents disparaissent. Cependant, sous l'effet du couplage avec la radiofréquence, de nouveaux effets d'interférence peuvent apparaître. Ainsi la diffusion $|\mathbf{k} \lambda\rangle \rightarrow\left|\mathbf{k}^{\prime} \lambda^{\prime}\right\rangle$ peut s'effectuer par les deux chemins $\left(D_{6} \alpha\right)$ et $\left(D_{6} \beta\right)$ qui ne sont « ouverts » tous

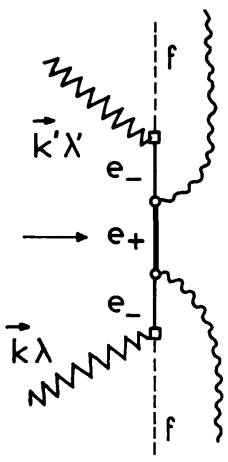

$\alpha)$

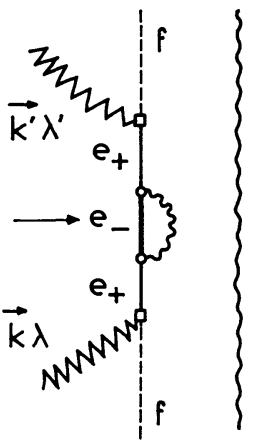

ß)
Diagramme $\mathrm{D}_{6}$. les deux que si les deux polarisations $\mathbf{e}_{\lambda}$ et $\mathbf{e}_{\lambda^{\prime}}$ sont cohérentes. A ces deux chemins qui correspondent au même état initial $|f n \mathbf{k} \lambda\rangle$ et au même état final $\left|f n \mathbf{k}^{\prime} \lambda^{\prime}\right\rangle$ correspondent deux amplitudes de diffusion $A_{a}$ et $A_{b}$ qui vont interférer. La transition vers l'état $\left|e_{+}, n-1\right\rangle$ marquée par une flèche sur $\left(D_{6} \alpha\right)$ sera réelle si :

$$
E_{k}+\omega=E_{0}+\frac{\omega_{0}}{2} \quad(\grave{a} \Gamma \text { près }) .
$$

De même, la transition vers l'état $\left|e_{-}, n+1\right\rangle$ marquée par une flèche sur $\left(D_{6} \beta\right)$ sera réelle si :

$$
\left.E_{k}+\omega=E_{0}-\frac{\omega_{0}}{2}+2 \omega \quad \text { (à } \Gamma \text { près }\right) \text {. }
$$

Les deux amplitudes de diffusion $A_{a}$ et $A_{b}$ seront donc simultanément importantes si :

a) $E_{k}=E_{0}$

b) $\omega_{0}=2 \omega$;

Le terme d'interférence entre $A_{a}$ et $A_{b}$ est donc résonnant au point de croisement pair $I_{n-1}^{2}$.

On peut imaginer aisément des paires de diagrammes d'ordre plus élevé donnant naissance à des termes d'interférence résonnants pour $\omega_{0}=2 p \omega$ (cf. diagrammes $\left(D_{7} \alpha\right)$ et $\left(D_{7} \beta\right)$ où $p=2$ ) (on vérifiera toutefois aisément que de tels diagrammes nécessitent

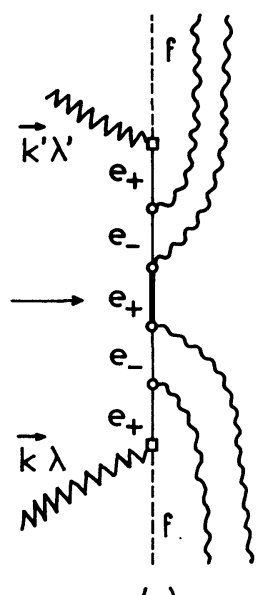

$\alpha)$

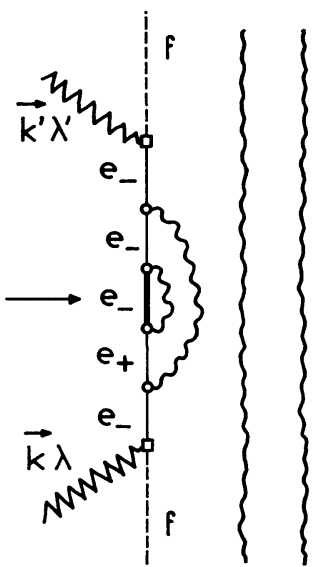

ß)
DiagrammE $D_{7}$.

une polarisation linéaire $\sigma$ du champ de radiofréquence alors que $\left(D_{6} \alpha\right)$ et $\left(D_{6} \beta\right)$ existent également avec une polarisation $\sigma^{+}$).

Nous voyons ainsi apparaître des résonances sur la section efficace de diffusion, liées à des interférences entre deux diagrammes comportant chacun un état intermédiaire résonnant et décrivant l'absorption réelle d'un photon optique et d'un ou plusieurs photons de radiofréquence (on voit en effet sur les diagrammes $\left(D_{6}\right)$ et $\left(D_{7}\right)$ que l'état intermédiaire résonnant n'est pas celui qui suit immédiatement l'absorption du photon optique incident). Les deux états intermédiaires simul- 
tanément résonnants sont ceux d'un croisement pair de $H_{0}$ pour une polarisation $\sigma$ de la radiofréquence. N'étant connectés à aucun ordre par $V$, ils ne peuvent apparaître que sur deux diagrammes différents, les deux chemins qui interfèrent n'étant simultanément ouverts que si les polarisations des photons optiques incidents et diffusés sont cohérentes $\left({ }^{6}\right)$. Notons la différence fondamentale avec les transitions à plusieurs quanta étudiées plus haut où les deux états résonnants figurent sur le même diagramme.

II. Etude quantitative. - Dans ce qui précède, nous avons décrit les phénomènes de façon intuitive et à l'ordre le plus bas où ils apparaissent. Nous nous proposons à présent de justifier et de préciser quantitativement les images qualitatives du paragraphe précédent : il nous faut notamment montrer que les effets résonnants décrits sont bien dus uniquement aux processus que nous avons indiqués, et d'autre part tenir compte des corrections apportées par tous les processus de diffusion d'ordre supérieur.

A. LA matrige de transition $T$. - Les sections efficaces de diffusion, et par suite les divers signaux mesurés, se calculent à partir de la matrice $S$ de la théorie des collisions [13], ou, ce qui revient au même, de la matrice $T$ définie par :

$$
S=I-2 \pi i \delta\left(E_{i}-E_{d}\right) T
$$

$E_{i}$ et $E_{d}$ étant les énergies des états initial $|f n \mathbf{k} \lambda\rangle$ et final $\left|f n^{\prime} \mathbf{k}^{\prime} \lambda^{\prime}\right\rangle$ de la diffusion. Tout le problème consiste donc à calculer les amplitudes de transition $\left\langle f n^{\prime} \mathbf{k}^{\prime} \lambda^{\prime}|T| f n \mathbf{k} \boldsymbol{\lambda}\right\rangle$. $T$ est donné par [13] :

$$
T=\mathscr{H}_{\mathrm{I}} \frac{1}{E_{i}-\mathscr{H}+i \varepsilon} \mathscr{H}_{\mathrm{I}}
$$

où $\mathscr{H}$ représente le hamiltonien total :

$$
\mathscr{H}=\mathscr{H}_{0}+\mathscr{H}_{\text {opt }}+V+\mathscr{H}_{\mathrm{I}}
$$

$T$ se présente ainsi comme le produit de trois termes décrivant successivement de droite à gauche :

a) L'absorption du photon incident et l'excitation optique de l'atome $\left(\mathscr{H}_{\mathrm{I}}\right)$;

b) L'évolution de l'atome dans l'état excité sous l'effet simultané des couplages $V$ et $\mathscr{H}_{\mathrm{I}}$ avec le champ de radiofréquence et le champ optique $\left(\frac{1}{E_{i}-\mathscr{H}+i \varepsilon}\right)$;

c) La retombée de l'atome dans l'état fondamental et l'émission du photon diffusé $\left(\mathscr{H}_{\mathrm{I}}\right)$.

(Nous négligerons dans toute la suite le processus antirésonnant qui correspond à l'émission du photon

(6) Il est en effet indispensable que le premier (dernier) état intermédiaire de diffusion soit différent sur les deux diagrammes (donc que les polarisations optiques soient cohérentes), car il est impossible d'atteindre à partir d'un même état par des transitions de radiofréquence les deux niveaux résonnants qui ne sont couplés entre eux à aucun ordre. diffusé $\left|\mathbf{k}^{\prime} \lambda^{\prime}\right\rangle$ précédant l'absorption du photon incident $|\mathbf{k} \lambda\rangle$.)

Le couplage $\mathscr{H}_{\mathrm{I}}$ avec le champ optique dans l'état excité est responsable des effets radiatifs bien connus : largeur naturelle $\Gamma$ et « self-énergie » $\Delta E$ du niveau excité. La théorie de l'émission spontanée [14] permet de montrer qu'on tient compte de ces effets en introduisant à la place de $\mathscr{H}_{\mathrm{I}}$ au dénominateur de $T$ dans (II.A.2) le terme complexe $\Delta E-i \frac{\Gamma}{2}$. (On admet que le couplage de l'atome avec la radiofréquence ne modifie pas le processus de l'émission spontanée, ce qui est parfaitement justifié car la radiofréquence n'a pas le temps de se coupler au système atomique pendant la durée très courte des phénomènes transitoires liés à l'émission spontanée d'un photon optique.)

On a donc :

$$
T=\mathscr{H}_{\mathrm{I}} G(z) \mathscr{H}_{\mathrm{I}}
$$

en introduisant le propagateur :

$$
G(z)=\frac{1}{z-\mathscr{H}_{0}-V}
$$

avec $z=E_{i}+i \frac{\Gamma}{2} \quad$ la self-énergie $\Delta E$ est réintégrée dans $\left.\mathscr{H}_{0}\right)$.

Le propagateur $G(z)$ peut se développer en puissances de $V$ :

$G=G_{0}+G_{0} V G_{0}+\ldots+G_{0}\left(V G_{0}\right)^{q}+\ldots$

avec :

$$
G_{0}=\frac{1}{z-\mathscr{H}_{0}} .
$$

On obtient finalement, en reportant (II.A.6) dans (II.A.4), le développement en série de perturbations de la matrice de transition :

$$
\begin{aligned}
T=\mathscr{H}_{\mathrm{I}} G_{0} \mathscr{H}_{\mathrm{I}}+ & \mathscr{H}_{\mathrm{I}} G_{0} V G_{0} \mathscr{H}_{\mathrm{I}}+\ldots \\
& +\mathscr{H}_{\mathrm{I}} G_{0}\left(V G_{0}\right)^{a} \mathscr{H}_{\mathrm{I}}+\ldots
\end{aligned}
$$

Les contributions de chaque terme de (II.A.8) à l'amplitude de diffusion peuvent être représentées par des diagrammes du type de ceux introduits plus haut et qui permettent de visualiser les divers processus physiques impliqués. Réciproquement, chaque diagramme correspond de façon univoque à un terme algébrique du développement de $T$, tout vertex étant associé à un élément de matrice de $V$ ou $\mathscr{H}_{\mathrm{I}}$, tout état intermédiaire à l'élément de matrice diagonal correspondant du propagateur $G_{0}$. Par exemple, au diagramme $\left(\mathrm{D}_{\mathbf{1}}\right)$ est associée l'amplitude :

$$
\begin{array}{r}
\left\langle f \mathbf{k}^{\prime} \lambda^{\prime}\left|\mathscr{H}_{\mathrm{I}}\right| e_{m}\right\rangle \frac{1}{E_{k}-E_{0}-m \omega_{0}+i \frac{\Gamma}{2}} \\
\left\langle e_{m}\left|\mathscr{H}_{\mathbf{I}}\right| f \mathbf{k} \lambda\right\rangle .
\end{array}
$$

Enfin, par suite de l'indiscernabilité des photons de radiofréquence qui appartiennent tous au même mode, 


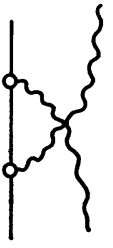

$\alpha)$

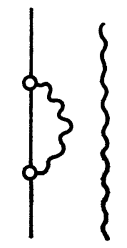

ß)
DIAGRAmme $\mathrm{D}_{8}$.

deux diagrammes qui ne diffèrent que par l'échange de photons de radiofréquence (par exemple $\left(D_{8} \alpha\right)$ et $\left(D_{8} \beta\right)$ ) correspondent au même processus physique. Il faut en garder un seul (celui par exemple où les deux lignes de photons ne se croisent pas).

B. Resommation des diagrammes. - On voit, d'après (II.A.8), qu'entre l'absorption du photon optique incident et l'émission du photon diffusé l'atome peut subir $0,1,2, \ldots, q \ldots$ interactions avec la radiofréquence. L'amplitude $\left\langle f n^{\prime} \mathbf{k}^{\prime} \lambda^{\prime}|T| f n \mathbf{k} \lambda\right\rangle$ s'obtient en sommant les contributions de tous ces processus.

Fixons l'énergie du photon optique incident. Nous remarquons que cette énergie ne peut coïncider qu'avec celle d'un petit nombre d'états intermédiaires de diffusion : par exemple, dans le cas d'un spin $J=1 / 2$, nous avons au plus deux états résonnants $a$ et $b$ aux points de croisement de $\mathscr{H}_{0}$ (cf. fig. 1). Ce sont évidemment ces états qui apportent dans chaque diagramme la contribution la plus importante à l'amplitude de transition. On a donc intérêt à distinguer ces niveaux de tous les autres dans la resommation. Dans ce but, nous introduisons les projecteurs $P$ et $Q$, projetant respectivement dans et hors de la multiplicité des niveaux intermédiaires résonnants et nous allons établir une nouvelle expression de $T$ qui particularise ces états résonnants.

1. Resommation formelle des diagrammes. - Nous représenterons la diffusion la plus générale par la série de diagrammes $\left(\mathrm{D}_{9}\right)$ dans lesquels nous avons indiqué en pointillé les niveaux initial et final $i$ et $d$ de la diffusion, en traits pleins les propagateurs des niveaux intermé-

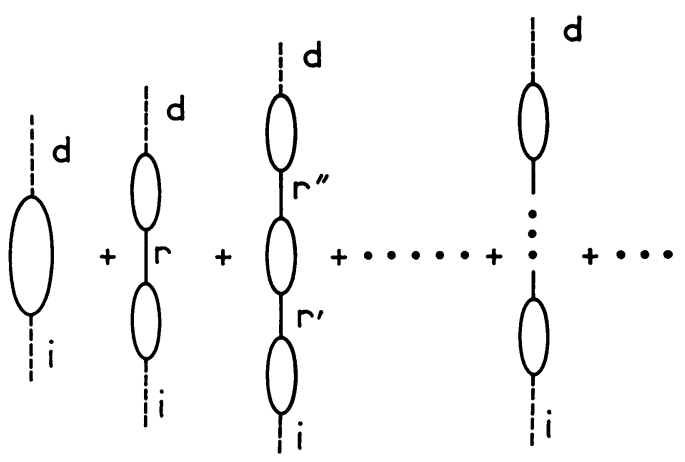

DIAGRAMME $\mathrm{D}_{9}$. diaires résonnants $r$; les figures ovales décrivent une diffusion soit directe, soit indirecte par l'intermédiaire de niveaux non résonnants, entre les états qu'elles relient.

Ainsi, le premier terme de $\left(D_{9}\right)$ ne comporte aucun niveau résonnant, les termes suivants contiennent successivement $1,2, \ldots, p \ldots$ états intermédiaires où l'énergie est conservée. Nous avons ainsi effectué un réarrangement des termes du développement (II.A.8) dans lequel nous avons regroupé ensemble tous les processus où le système subit un nombre donné de transitions intermédiaires réelles.

A chaque partie des diagrammes $\left(\mathrm{D}_{9}\right)$ correspond une expression algébrique bien définie : aux propagateurs des niveaux résonnants (traits pleins) correspondent les éléments de matrice de $P G_{0} P$; à chacune des figures ovales (vertex généralisé) est associée une série de termes décrivant une diffusion directe ou indirecte via $1,2, \ldots, q \ldots$ niveaux tous non résonnants :

$$
\begin{gathered}
W+W Q G_{0} Q W+W Q G_{0} Q V Q G_{0} Q W+\ldots \\
+W Q G_{0} Q\left(V Q G_{0} Q\right)^{q} W+\ldots
\end{gathered}
$$

$W$ est mis pour $\mathscr{H}_{\mathrm{I}}$ ou $V$ suivant que l'on a, à l'extrémité de la chaîne correspondante, l'état initial ou final $i$ ou $d$, ou un état résonnant $r$. La présence du projecteur $Q$ assure que l'on ne passe jamais par un niveau résonnant.

(II.B.1) se resomme formellement pour donner :

$$
W+W K W=W(1+K W)=(1+W K) W
$$

avec :

$$
K=\frac{Q}{z-\mathscr{H}_{0}-Q V Q} .
$$

Le développement de $K$ en puissances de $V$ porté dans (II.B.2) redonne bien en effet (II.B.1). En factorisant la partie centrale des diagrammes comprise entre la première et la dernière apparition d'un niveau résonnant au cours de la diffusion, nous pouvons mettre $\left(\mathrm{D}_{9}\right)$ sous la forme $\left(\mathrm{D}_{10}\right)$.

Dans le premier terme de $\left(\mathrm{D}_{10}\right)$, l'ovale relie $i$ et $d$. Il faut donc, dans (II.B.2), remplacer $W$ par $\mathscr{H}_{\mathrm{I}}$, et l'on a :

$$
\bigcap_{i}^{d}=\mathscr{H}_{\mathrm{I}}+\mathscr{H}_{\mathrm{I}} K \mathscr{H}_{\mathrm{I}}=\mathscr{H}_{\mathrm{I}} K \mathscr{H}_{\mathrm{I}}
$$

(en effet, $\mathscr{H}_{\mathrm{I}}$ ne peut connecter $i$ et $d$ ).

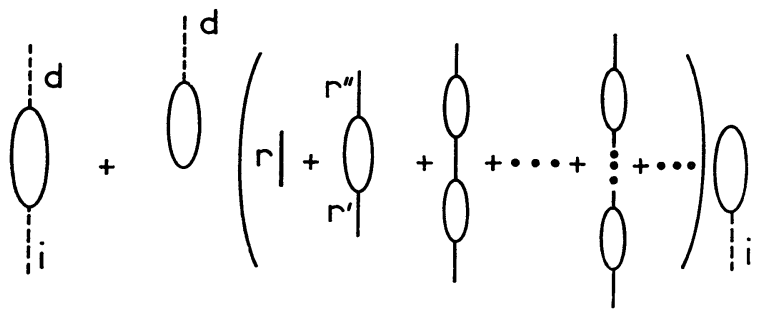

Diagramme $\mathrm{D}_{\mathbf{1 0}}$. 
Les deux ovales qui figurent à l'extérieur de la parenthèse de $\left(\mathrm{D}_{10}\right)$ relient $i$ ou $d$ et un état résonnant. On a donc, d'après (II.B.2) :

$$
\bigcap_{i}=[1+V K] \mathscr{H}_{\mathrm{I}}=F \mathscr{H}_{\mathrm{I}}
$$

en posant :

$$
F=1+V K .
$$

De même, toujours d'après (II.B.2) :

$$
\overbrace{}^{d}=\mathscr{H}_{\mathrm{I}}[1+K V]=\mathscr{H}_{\mathrm{I}} F^{\prime}
$$

en posant :

$$
F^{\prime}=1+K V
$$

Enfin, tous les ovales figurant à l'intérieur de la parenthèse de $\left(D_{10}\right)$ relient deux états résonnants de sorte que l'expression algébrique correspondante est (II.B.2) où l'on remplace $W$ par $V$, c'est-à-dire encore :

$$
R=V+V K V .
$$

Il s'ensuit que l'expression algébrique correspondant à la parenthèse de $\left(D_{10}\right)$ s'écrit :

$P G_{0} P+P G_{0} P R P G_{0} P+P G_{0} P R P G_{0} P R P G_{0} P+\ldots$

expression qui se resomme pour donner :

$$
\frac{P}{z-\mathscr{H}_{0}-P R P}
$$

ce qui, d'ailleurs, n'est autre que la projection $P G P \mathrm{du}$ propagateur " perturbé » $G(z)=\frac{1}{z-\mathscr{H}_{0}-V}$ dans la multiplicité des états résonnants. En effet, on peut manifestement développer $P G(z) P$ en puissance de $V$ comme dans (II.A.6) et regrouper les termes en particularisant les états résonnants comme cela est fait dans la parenthèse de $\left(\mathrm{D}_{10}\right)$.

Finalement, l'expression algébrique de $T$ associée à $\left(D_{10}\right)$ et obtenue en particularisant les états résonnants s'écrit :

$$
T=\mathscr{H}_{\mathrm{I}} K \mathscr{H}_{\mathrm{I}}+\mathscr{H}_{\mathrm{I}} F^{\prime} \frac{P}{z-\mathscr{H}_{0}-P R P} F \mathscr{H}_{\mathrm{I}}
$$

où $K, F, F^{\prime}, R$ sont donnés par (II.B.3), (II.B.6), (II.B.8), (II.B.9).

Nous avons donné de (II.B.11) une démonstration purement diagrammatique. On peut en donner une démonstration purement algébrique (cf. appendice 2 ), qui a l'avantage d'être plus rapide, mais l'inconvénient d'être moins physique.

2. Approximations sur la formule resommée. - Le développement en série de l'opérateur $K$ et celui des opérateurs $F, F^{\prime}$ et $R$ qui s'expriment à l'aide de $K$ sont très rapidement convergents tant que les éléments de matrice de $V$ sont très petits devant $\omega$, car aucun de leurs dénominateurs d'énergie n'est résonnant. Il en résulte que l'on peut remplacer, dans l'expression (II.B.11) de $T$, les éléments de matrice de ces opérateurs par leur expression à l'ordre le plus bas où ils sont non nuls.

D'autre part, ces opérateurs sont en toute rigueur des fonctions de la variable $z=E_{i}+i \frac{\Gamma}{2}$, mais ce sont en fait des fonctions très lentement variables au voisinage d'un croisement de niveaux de $\mathscr{H}_{0}$, car aucun des dénominateurs d'énergie ne peut diverger; on peut donc négliger cette variation ainsi que le terme $i \frac{\Gamma}{2}$ et remplacer $z$ par la valeur fixe $\bar{E}$ de l'énergie au point de croisement des niveaux. On appellera donc $\bar{K}, \bar{F}, \bar{F}^{\prime}$ et $\bar{R}$ l'expression à l'ordre le plus bas, pour la valeur $z=\bar{E}$, des opérateurs $K$, $F, F^{\prime}$ et $R$, et on remplacera $T$ par l'expression approchée :

$$
\bar{T}=\mathscr{H}_{\mathrm{I}} \bar{K} \mathscr{H}_{\mathrm{I}}+\mathscr{H}_{\mathrm{I}} \bar{F}^{\prime} \frac{P}{z-\mathscr{H}_{0}-P \bar{R} P} \bar{F} \mathscr{H}_{\mathrm{I}} .
$$

Remarquons bien qu'une telle approximation est nettement meilleure que celle que l'on fait en calculant $T$ à un certain ordre de perturbation en $V$ selon (II.A.8). On obtient en effet $\bar{T}$ en remplaçant dans $\left(\mathrm{D}_{9}\right)$ chacune des figures ovales par son expression à l'ordre le plus bas (avec $z=\bar{E}$ ) représentée dans $\left(\mathrm{D}_{11}\right)$ par un rec-

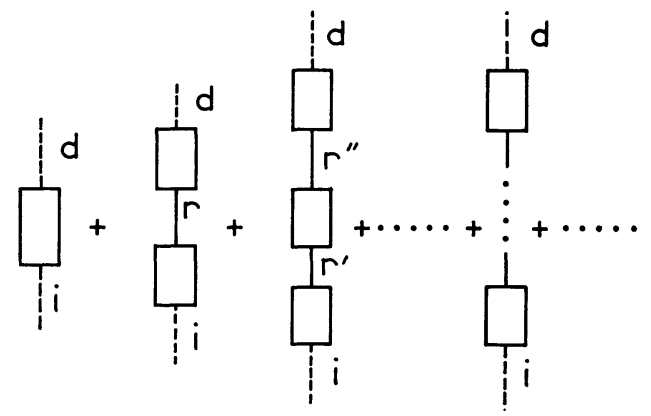

DIAGRAMme $\mathrm{D}_{11}$.

tangle. Ce faisant, on resomme cependant une série infinie de diagrammes qui font intervenir à tous les ordres les niveaux résonnants. On conçoit aisément qu'en traitant ainsi de façon complète l'effet prépondérant de toutes les transitions réelles et à l'ordre le plus bas les effets très faibles des transitions virtuelles, on fait une approximation très raisonnable.

3. Règle de calcul de l'amplitude de transition. - L'amplitude de transition $\left\langle f n^{\prime} \mathbf{k}^{\prime} \lambda^{\prime}|\bar{T}| f n \mathbf{k} \lambda\right\rangle$ calculée à partir de (II.B.12) est une somme d'un certain 
nombre d'amplitudes partielles qui correspondent aux différents éléments de matrice de :

$$
P \bar{G} P=\frac{P}{z-\mathscr{H}_{0}-P \bar{R} P}
$$

à l'intérieur de la multiplicité des niveaux résonnants $\left(r, r^{\prime} \ldots\right)$.

(II.B.12) s'écrit en effet :

$\bar{T}=\mathscr{H}_{\mathrm{I}} \bar{K} \mathscr{H}_{\mathrm{I}}+\sum_{r r^{\prime}} \mathscr{H}_{\mathrm{I}} \bar{F}^{\prime}|r\rangle\left\langle r|\bar{G}| r^{\prime}\right\rangle\left\langle r^{\prime}\right| \bar{F} \mathscr{H}_{\mathrm{I}}$.

La règle suivante permet de calculer les différents termes de (II.B.13) à partir de diagrammes à l'ordre le plus bas.

a) On commence par déterminer tous les diagrammes à l'ordre le plus bas reliant $|f n \mathbf{k} \lambda\rangle$ à $\left|f n^{\prime} \mathbf{k}^{\prime} \lambda^{\prime}\right\rangle$ et contenant soit aucun, soit un, soit deux états intermédiaires résonnants différents.

b) On conserve tel quel le diagramme non résonnant, ce qui donne le premier terme de (II.B.13).

c) Dans chacun des autres diagrammes, on garde les propagateurs des chaînes extrêmes non résonnantes; pour le diagramme contenant un seul état intermédiaire résonnant $r$, on remplace la partie centrale (propagateur $\left\langle r\left|G_{0}\right| r\right\rangle$ de l'état $r$ ) par l'élément diagonal de $\bar{G},\langle r|\bar{G}| r\rangle$ (on obtient ainsi tous les termes du deuxième membre de (II.B.13) correspondant à $r=r^{\prime}$ ); pour le diagramme contenant deux états intermédiaires résonnants différents $r, r^{\prime}$, on remplace la partie centrale ou " chaîne »

$$
\left\langle r\left|G_{0} P \bar{R} P G_{0}\right| r^{\prime}\right\rangle
$$

reliant $r$ et $r^{\prime}$ par $\left\langle r|\bar{G}| r^{\prime}\right\rangle$ (ce qui donne tous les autres termes de (II.B.13)) : en d'autres termes, nous effectuons sur les diagrammes à l'ordre le plus bas une renormalisation du propagateur de la partie résonnante à l'aide des éléments de matrice de $P \bar{G} P$. Illustrons cette renormalisation par deux exemples empruntés au cas d'un spin $J=1 / 2$ et d'un champ de radiofréquence $\sigma$ et relatifs au cas d'un croisement pair et d'un croisement impair de niveaux (cf. fig. 1) :

- Croisement pair : Les niveaux qui se croisent ne sont couplés à aucun ordre par $V$. L'opérateur $P \bar{G} P$ est diagonal et un diagramme ne peut passer que par l'un des deux niveaux résonnants à la fois. Celui qui passe par le niveau $a$, à l'ordre le plus bas, correspond à l'amplitude :

$$
\begin{aligned}
& A_{a}=\left\langle f n^{\prime} \mathbf{k}^{\prime} \lambda^{\prime}\left|\mathscr{H}_{\mathrm{I}} \bar{F}^{\prime}\right| a\right\rangle \frac{1}{E_{i}-E_{a}+i \frac{\Gamma}{2}} \\
&\left\langle a\left|\bar{F} \mathscr{H}_{\mathrm{I}}\right| f n \mathbf{k} \lambda\right\rangle .
\end{aligned}
$$

La règle de resommation revient à remplacer 1

$\frac{1}{E_{i}-E_{a}+i \frac{\Gamma}{2}}$ par l'élément de matrice diagonal $\bar{G}_{a a}=\frac{1}{E_{i}-E_{a}-\overline{R_{a a}}+i \frac{\Gamma}{2}}$. L'amplitude devient :

$$
\begin{aligned}
A_{a}^{\prime}=\left\langle f n^{\prime} \mathbf{k}^{\prime} \lambda^{\prime}\left|\mathscr{H}_{\mathrm{I}} \bar{F}^{\prime}\right| a\right\rangle & \frac{1}{E_{i}-E_{a}-\overline{R_{a a}}+i \frac{\Gamma}{2}} \\
& \left\langle a\left|\bar{F} \mathscr{H}_{\mathrm{I}}\right| f n \mathbf{k} \boldsymbol{\lambda}\right\rangle . \quad \text { II } . \mathrm{B}
\end{aligned}
$$

Sous l'effet des transitions virtuelles décrites par $\overline{R_{a a}}$, le propagateur $G_{0 a a}$ du niveau résonnant a été renormalisé en $\overline{G_{a a}}$, ce qui a pour effet de déplacer d'une quantité du second ordre en $V$ l'énergie $E_{a}$ de ce niveau.

- Croisement impair : Les niveaux $a$ et $b$ qui se croisent sont couplés par $V$. L'opérateur $P \bar{G} P$ a des éléments de matrice non diagonaux : en inversant $z-\mathscr{H}_{0}-P \bar{R} P$ (qui est dans ce cas simple une matrice $2 \times 2$ ), on obtient sans difficulté :

$$
\overline{G_{b a}}(z)=\frac{\overline{R_{b a}}}{\left(z-E_{a}-\overline{R_{a a}}\right)\left(z-E_{b}-\overline{R_{b b}}\right)-\left|\overline{R_{a b}}\right|^{2}} .
$$

Il existe donc des diagrammes passant successivement par les deux niveaux résonnants $a$ et $b$, le diagramme à l'ordre le plus bas correspondant à l'amplitude de transition :

$$
A_{b a}=\left\langle f n^{\prime} \mathbf{k}^{\prime} \lambda^{\prime}\left|\mathscr{H}_{\mathrm{I}} \bar{F}^{\prime}\right| b\right\rangle\left[\frac{1}{E_{i}-E_{b}+i \frac{\Gamma}{2}} \overline{R_{b a}} \frac{1}{E_{i}-E_{a}+i \frac{\Gamma}{2}}\right]\left\langle a\left|\bar{F} \mathscr{H}_{\mathrm{I}}\right| f n \mathbf{k} \lambda\right\rangle .
$$

La règle de resommation conduit à remplacer la partie résonnante entre crochets de (II.B.17) par $\overline{G_{b a}}(z)$. On obtient alors :

$$
A_{b a}^{\prime}=\left\langle f n^{\prime} \mathbf{k}^{\prime} \lambda^{\prime}\left|\mathscr{H}_{\mathrm{I}} \bar{F}^{\prime}\right| b\right\rangle \frac{\overline{R_{b a}}}{\left(E_{i}-E_{a}-\overline{R_{a a}}+i \frac{\Gamma}{2}\right)\left(E_{i}-E_{b}-\overline{R_{b b}}+i \frac{\Gamma}{2}\right)-\left|\overline{R_{a b}}\right|^{2}}\left\langle a\left|\bar{F} \mathscr{H}_{\mathrm{I}}\right| f n \mathbf{k} \lambda\right\rangle .
$$


La resommation modifie donc, dans ce cas, profondément la structure des dénominateurs d'énergie résonnants. Nous verrons que cette modification correspond non seulement à un déplacement, mais aussi à un élargissement des raies de résonance magnétique.

C. Calgul des signaux de RÉsonance magnétique. - 1. Expression du signal optique mesuré. - La probabilité par unité de temps $W f n \mathbf{k} \lambda \rightarrow f n^{\prime} \mathbf{k}^{\prime} \lambda^{\prime}$ de la

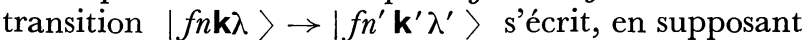
différentes les directions de $\mathbf{k}$ et de $\mathbf{k}^{\prime}$ :

$$
\begin{gathered}
W f n \mathbf{k} \lambda, f n^{\prime} \mathbf{k}^{\prime} \lambda^{\prime}=2 \pi\left|\left\langle f n^{\prime} \mathbf{k}^{\prime} \lambda^{\prime}|T| f n \mathbf{k} \lambda\right\rangle\right|^{2} \\
\delta\left(\left(n-n^{\prime}\right) \omega+E_{k}-E_{k^{\prime}}\right) .
\end{gathered}
$$

Comme nous ne détectons pas les photons de radiofréquence et que nous n'analysons pas l'énergie des photons optiques diffusés, mais seulement leur direction et leur état de polarisation, nous devons sommer la probabilité de transition sur tous les états finaux correspondant à des nombres $n^{\prime}$ de photons de radiofréquence différents et à des énergies $E_{k^{\prime}}$ différentes. D'autre part, l'onde optique incidente n'est pas monochromatique : on peut la considérer comme constituée par un mélange statistique de photons $|\mathbf{k} \lambda\rangle$ dont la répartition en énergie est donnée par une fonction $u\left(E_{k}\right)$ centrée autourd'une valeur $E_{k}$ de largeur $\Delta(f g .2)$.

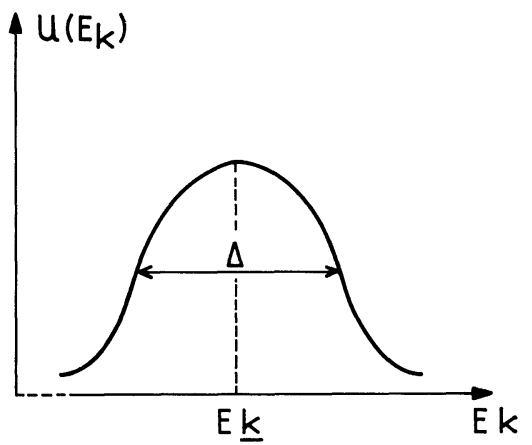

FIG. 2.

La probabilité de diffusion de cette onde s'obtiendra en pondérant la probabilité (II.C.1) par la forme de raie. Finalement, la section efficace de diffusion dans la direction $\mathbf{k}^{\prime}$ avec la polarisation $\mathbf{e}_{\lambda^{\prime}}$ s'écrira $\left({ }^{7}\right)$ :

$$
\begin{aligned}
& \mathscr{S}_{\mathbf{k} \lambda \rightarrow \mathbf{k}^{\prime} \lambda^{\prime}}=\text { Cte } \sum_{n^{\prime}} \iint u\left(E_{k}\right) \mathrm{d} E_{k} \mathrm{~d} E_{k^{\prime}} \\
& \quad \delta\left[E_{k}-E_{k^{\prime}}+\left(n-n^{\prime}\right) \omega\right]\left|\left\langle f n^{\prime} \mathbf{k}^{\prime} \lambda^{\prime}|T| f n \mathbf{k} \lambda\right\rangle\right|^{2} .
\end{aligned}
$$

Nous supposerons, dans la suite de cet article, que l'excitation optique s'effectue en raie large (« broadline ») :

$$
\Delta \gg \omega_{0}, \omega,\left|\gamma H_{1}\right|, \Gamma \text {. }
$$

( ${ }^{7}$ Les formules (II.C.2) et (II.C.4) ne sont valables en toute rigueur que si l'état fondamental est diamagnétique (voir note $\left({ }^{3}\right)$ ). Il est facile de les généraliser à un cas où l'état fondamental est paramagnétique.
En d'autres termes, le passage du train d'onde sur l'atome s'effectue en un temps $1 / \Delta$ très court devant tous les temps d'évolution propre du système atomique couplé à la radiofréquence. Cette hypothèse est parfaitement justifiée dans le cas où l'irradiation optique est réalisée avec une source ordinaire (nous réservons à un prochain article l'étude du cas inverse où la largeur $\Delta$ est très faible (excitation " narrow-line »)).

Nous pouvons alors, dans (II.C.2), remplacer la fonction $u\left(E_{k}\right)$ par l'unité, et la section efficace «broadline » devient :

$$
\begin{aligned}
& \mathscr{S}_{\mathbf{k} \lambda \rightarrow \mathbf{k}^{\prime} \lambda^{\prime}}=\text { Cte } \sum_{n^{\prime}} \iint \mathrm{d} E_{k} \mathrm{~d} E_{k^{\prime}} \\
& \quad \delta\left[E_{k}-E_{k^{\prime}}+\left(n-n^{\prime}\right) \omega\right]\left|\left\langle f n^{\prime} \mathbf{k}^{\prime} \lambda^{\prime}|T| f n \mathbf{k} \boldsymbol{\lambda}\right\rangle\right|^{2} .
\end{aligned}
$$

Remarquons enfin que nous avons supposé que l'état initial de la radiofréquence est un état $|n\rangle$ incohérent. Nous aborderons en appendice 4 le cas d'un champ de radiofréquence cohérent et l'étude des modulations de la section efficace de diffusion optique.

2. Transitions réelles à plusieurs quanta (champ de radiofréquence en polarisation $\sigma)$. - Plaçons-nous dans les conditions du $\S$ I.C (excitation en $\sigma^{-}$, détection en $\sigma^{+}$; seuls $B_{-}$et $B_{+}^{\prime}$ sont non nuls) et au voisinage d'un point de croisement impair $\omega_{0}=(2 p+1) \omega$.

D'après (II.C.4), le signal observé est la somme d'un certain nombre de termes correspondant chacun à un nombre donné $n^{\prime}$ de photons de radiofréquence dans l'état final de diffusion et à une énergie donnée $E_{k}$ du photon optique incident. Chacun des termes de (II.C.4) est calculé à partir de la formule (II.B.12) (dont nous rappelons qu'elle correspond, bien qu'approchée, à la resommation d'une infinité de diagrammes). Nous allons faire l'inventaire de tous les termes de (II.C.4) et montrer que l'un d'entre eux est beaucoup plus grand que les autres.

a) Inventaire des diagrammes résonnants. - Supposons $n^{\prime}$ et $E_{k}$ fixés et appelons $|a\rangle$ et $|b\rangle$ les deux niveaux intermédiaires résonnants pour cette énergie du photon optique incident. $|a\rangle$ et $|b\rangle$ qui se croisent en

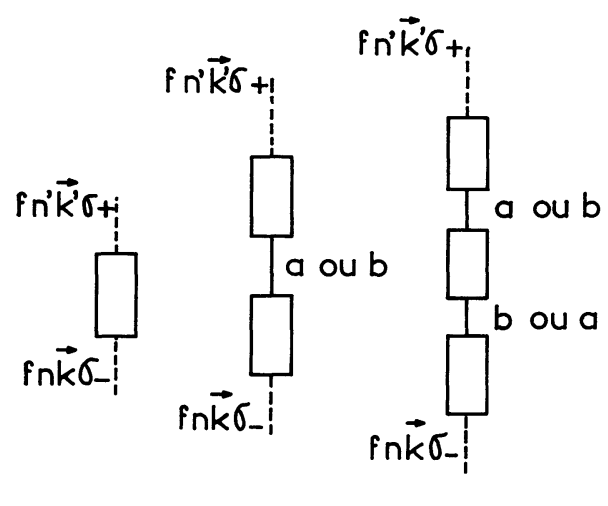
$\alpha)$
ß)
○)

DIAGRAmme $D_{12}$. 
$\omega_{0}=(2 p+1) \omega$ diffèrent de $(2 p+1)$ photons de radiofréquence. Conformément aux règles du $\S$ II.B.3, seuls interviennent dans le calcul de l'amplitude de transition les diagrammes $\left(D_{12} \alpha, \beta, \gamma\right)$ dans lesquels les rectangles représentent, comme dans $\left(D_{11}\right)$, l'expression à l'ordre le plus bas des chaînes d'états non résonnants reliant deux niveaux. La probabilité de transition fait intervenir le produit deux à deux des contributions de ces diagrammes. Chacun de ces produits n'est résonnant dans le champ $\omega_{0}=(2 p+1) \omega$ que s'il contient simultanément les propagateurs des deux états résonnants différents $|a\rangle$ et $|b\rangle$ qui se croisent dans ce champ (cf. § I.C). Nous devons donc tenir compte des quatre types de produits différents représentés diagrammatiquement dans $\left(\mathrm{D}_{13} \alpha, \beta, \gamma, \delta\right)$.

Étant donné que les premier et dernier états intermédiaires de diffusion sont identiques dans les deux diagrammes d'un produit (ce sont les états $\left|e_{-}, n\right\rangle$ et $\left.\left|e_{+}, n^{\prime}\right\rangle\right)$, on voit que dans chacun des produits

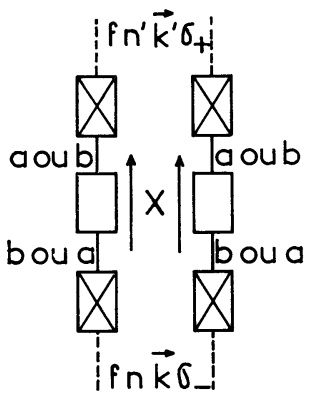

$\alpha)$

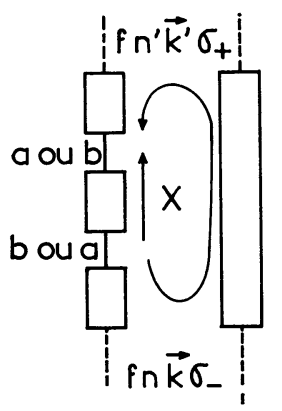

४)

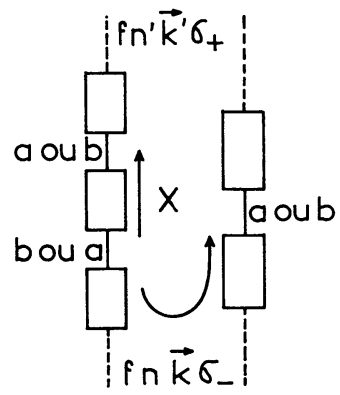

ß)

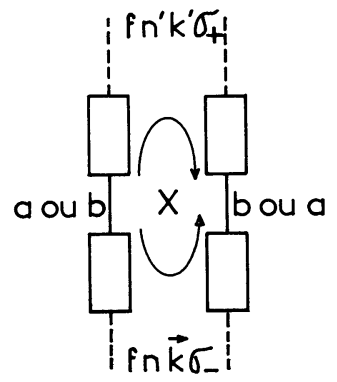

ठ)
DIAGRAMME $\mathrm{D}_{13}$. on peut mettre en évidence au moins deux « chaînes » d'états intermédiaires reliés les uns aux autres par l'interaction $V$ qui permettent de passer de $a$ à $b$ (ces « chaînes " sont symbolisées par des flèches sur $\left.\left(D_{13}\right)\right)$. Chacune de ces " chaînes » étant d'ordre au moins $2 p+1$, chaque produit a un ordre minimum $2(2 p+1)$. Nous allons voir cependant que, bien que les contributions des quatre produits $\alpha, \beta$, $\gamma, \delta$ puissent être toutes du même ordre en $V$, c'est la probabilité de transition associée à $\left(\mathrm{D}_{13} \alpha\right)$ qui l'emporte sur les autres : elle correspond en effet au carré d'un diagramme contenant deux transitions intermédiaires réelles et aura donc quatre dénominateurs d'énergie résonnants; par contre, les termes d'interférence $\left(D_{13} \beta, \gamma, \delta\right)$ ne contiennent chacun que deux ou trois niveaux résonnants et donnent donc des contributions d'un ordre $\Gamma / \omega$ ou $\Gamma^{2} / \omega^{2}$ fois plus petites que celle de $\left(D_{13} \alpha\right)$, donc totalement inobservables.

Finalement, nous ne conserverons donc que le terme $\left(D_{13} \alpha\right)$. Pour qu'il soit effectivement d'ordre minimum $2(2 p+1)$, il faut que les chaînes d'entrée et de sortie de ces diagrammes (marquées par des croix) soient d'ordre zéro en $V$ (autrement dit, se réduisent au simple vertex $\mathscr{H}_{\mathrm{I}}$ ). En effet, chacun des rectangles centraux de ces diagrammes est déjà d'ordre $2 p+1$. Cette condition revient à imposer au niveau suivant immédiatement l'absorption du photon optique incident $\left|e_{-}, n\right\rangle$ et au niveau précédant immédiatement l'émission du photon diffusé $\left|e_{+}, n^{\prime}\right\rangle$ d'être les niveaux résonnants $|a\rangle$ et $|b\rangle$. Le nombre de photons $n^{\prime}=n-(2 p+1)$ de l'état final est alors imposé ainsi que le croisement de niveaux $|a\rangle=\left|e_{-}, n\right\rangle,|b\rangle=\left|e_{+}, n-(2 p+1)\right\rangle$. Le seul diagramme qui intervient dans le calcul du signal de résonance est alors celui que nous avions envisagé dans la partie qualitative de notre étude (dans le cas de la transition $\omega_{0}=3 \omega$ ) qui se trouve ainsi justifiée. L'atome est excité optiquement de façon résonnante dans l'état $\left|e_{-}, n\right\rangle$, subit $(2 p+1)$ transitions de radiofréquence dont la dernière le conduit au niveau résonnant $\left|e_{+}, n-(2 p+1)\right\rangle$ d'où il émet le photon diffusé.

b) Calcul du signal. Interprétation physique. - L'amplitude de transition, renormalisée suivant les règles du $\S$ II.B.3, se déduit simplement de (II.B.18) en y faisant $\bar{F}=\bar{F}^{\prime}=P$ :

$$
\left\langle f, n-(2 p+1), \mathbf{k}^{\prime} \lambda^{\prime}|\bar{T}| f n \mathbf{k} \lambda\right\rangle=B_{+}^{\prime} \frac{\overline{R_{b a}}}{\left(E_{i}-E_{a}-\overline{R_{a a}}+i \frac{\Gamma}{2}\right)\left(E_{i}-E_{b}-\overline{R_{b b}}+i \frac{\Gamma}{2}\right)-\left|\overline{R_{a b}}\right|^{2}} B_{-} .
$$

Le signal de résonance magnétique $\mathscr{S}$ s'obtient alors en reportant (II.G.5) dans (II.C.4) et en intégrant sur les énergies $E_{k}$ par la méthode des résidus :

$$
\overline{\mathscr{S}}=\left|B_{+}^{\prime}\right|^{2} \frac{\left|\overline{R_{a b}}\right|^{2}}{\left(E_{a}-E_{b}+\overline{R_{a a}}-\overline{R_{b b}}\right)^{2}+\left|\overline{R_{a b}}\right|^{2}+\Gamma^{2}}\left|B_{-}\right|^{2} .
$$


L'interprétation physique de cette formule est très claire : le temps de passage $1 / \Delta$ du train d'onde incident sur le système atomique étant très court, les trois phases successives : excitation optique, évolution dans l'état excité, émission spontanée, sont indépendantes; l'atome absorbe " instantanément » la lumière incidente $\sigma_{-}$, ce qui le porte dans l'état non perturbé $|a\rangle=\left|e_{-}, n\right\rangle$, la probabilité de ce processus d'absorption étant proportionnelle à $\left|B_{-}\right|^{2}$; puis il évolue sous l'effet de la résonance magnétique de l'état $|a\rangle$ à l'état $|b\rangle=\left|e_{+}, n-(2 p+1)\right\rangle$, la probabilité de ce processus étant donnée par le terme central de (II.C.6); enfin, il émet « instantanément 》 le photon diffusé en retombant à l'état fondamental avec une probabilité proportionnelle à $\left|B_{+}^{\prime}\right|^{2}$ : (II.G.6) n'est autre que l'expression déjà obtenue par ailleurs [4], [15], [16] du signal de résonance magnétique détecté optiquement, la lumière ne jouant ici qu'un rôle de préparation et de détection de l'évolution du système.

Pour calculer explicitement les formes de raie des transitions à plusieurs quanta, nous devons nous placer au voisinage de chaque point de croisement :

$$
\omega_{0}=(2 p+1) \omega
$$

et calculer les quantités $\overline{R_{a b}}, \overline{R_{a a}}$ et $\overline{R_{b b}}$ qui s'expriment à partir de (II.B.9) en ne gardant que la partie non nulle à l'ordre le plus bas du développement de l'opérateur $K$. On obtient en général le profil de raie :

$$
\overline{\mathscr{S}}=\frac{I_{2 p+1}\left(\gamma H_{1}\right)^{2(2 p+1)}}{\left[\omega_{0}-(2 p+1) \omega+\delta_{2 p+1}\left(\gamma H_{1}\right)^{2}\right]^{2}+\mathrm{e}_{2 p+1}\left(\gamma H_{1}\right)^{2(2 p+1)}+\Gamma^{2}}
$$

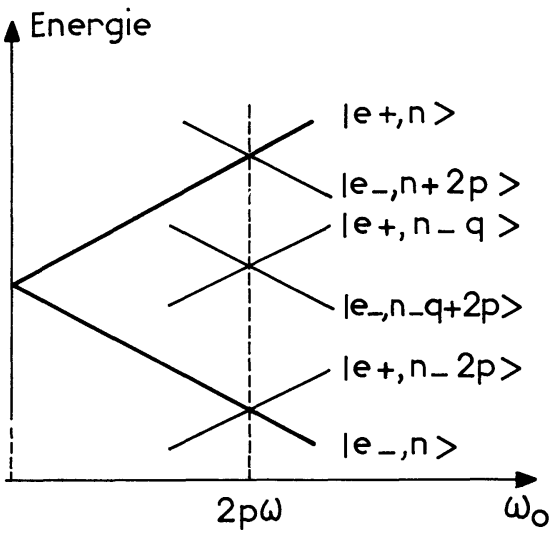

FIG. 3.

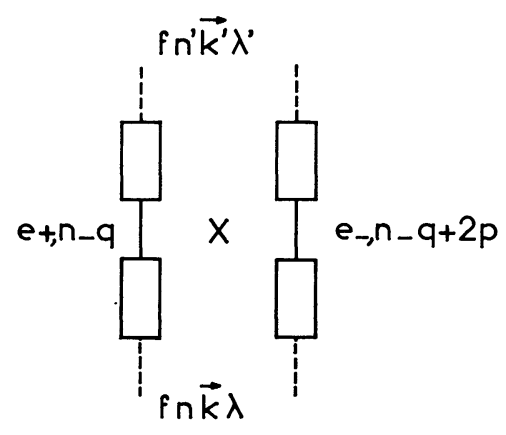

DIAGRAMme $\mathrm{D}_{14}$.
$I_{2 p+1}, \delta_{2 p+1}, e_{2 p+1}$ étant des constantes d'intensité, de déplacement et d'élargissement entièrement calculables.

La formule (II.C.7) résume toutes les propriétés des transitions à plusieurs quanta telles qu'elles ont été décrites par Winter [4] :

La résonance à $(2 p+1)$ quanta a une intensité proportionnelle à $H_{1}^{2(2 p+1)}$ (pour des valeurs suffisamment faibles de $H_{1}$ ), une largeur :

$$
\sqrt{\Gamma^{2}+\mathrm{e}_{2 p+1}\left(\gamma H_{1}\right)^{2(2 p+1)}}
$$

égale à $\Gamma$ pour des valeurs très petites de $H_{1}$, qui augmente avec $H_{1}$ et devient proportionnelle à $H_{1}^{2 p+1}$ lorsque $\left|\gamma H_{1}\right| \gg \Gamma$. Enfin, la résonance subit un déplacement radiatif de type Bloch-Siegert [17] en $H_{1}^{2}$ vers les faibles valeurs du champ égal à $-\delta_{2 p+1}\left(\gamma H_{1}\right)^{2}$; il est d'ailleurs possible de donner une expression générale du coefficient $\delta_{2 p+1}$ (cf. appendice 3 ). L'interprétation physique des déplacements et élargissements radiatifs des résonances est très claire : les déplacements (décrits par les termes $\overline{R_{a a}}$ et $\overline{R_{b b}}$ ) sont dus aux transitions virtuelles de radiofréquence qui déplacent les énergies des niveaux $|a\rangle$ et $|b\rangle$, les élargissements (décrits par le terme non diagonal $\left|R_{a b}\right|^{2}$ ) sont dus aux transitions réelles entre $|a\rangle$ et $|b\rangle$ qui raccourcissent en quelque sorte la durée de vie de ces deux niveaux.

3. Nouvelles raies de résonance magnétique (champ de radiofréquence en polarisation $\sigma$ ). - Rappelons rapidement les conditions d'observation de ces résonances : les polarisations optiques d'excitation et de détection sont « cohérentes » $\left(B_{+}, B_{-}, B_{+}^{\prime}, B_{-}^{\prime} \neq 0\right)$, le champ magnétique correspond à un croisement pair $\omega_{0}=2 p \omega$. Les niveaux qui se croisent dans ce champ ne sont alors couplés à aucun ordre et il ne peut donc apparaître qu'un niveau résonnant dans chaque diagramme. La résonance ne peut provenir que de l'interférence de deux diagrammes contenant chacun l'un des deux niveaux résonnants qui se croisent. Contrairement aux résultats du paragraphe précédent, 
nous allons voir que plusieurs termes contribuent à l'expression du signal résonnant :

a) Inventaire des diagrammes. - Nous appellerons $|a\rangle=\left|e_{+}, n-q\right\rangle$ et $|b\rangle=\left|e_{-}, n-q+2 p\right\rangle$ les niveaux résonnants pour une énergie donnée $E_{k}$ du photon optique incident. $q$ peut prendre toutes les valeurs algébriques positives, négatives ou nulles et permet de numéroter tous les croisements alignés dans le champ $\omega_{0}=2 p \omega$ (cf. fig. 3). La partie résonnante de la probabilité de transition se représente comme le produit des diagrammes $\left(\mathrm{D}_{14}\right)$.

L'ordre de perturbation de ce produit est égal à la somme des ordres en $V$ des quatre parties rectangulaires qu'il contient. Nous allons déterminer les conditions pour lesquelles cet ordre est minimum. Envisageons tout d'abord le produit des deux rectangles compris entre l'état initial de la diffusion et l'état résonnant $|a\rangle$ ou $|b\rangle$. L'ordre de ce produit est évidemment égal à $|q|+|2 p-q|$ et prend une valeur minimale égale à $2 p$ si la condition $0 \leqslant q \leqslant 2 p$ est remplie, autrement dit si le croisement des niveaux $|a\rangle$ et $|b\rangle$ a une énergie comprise entre celle des niveaux $\left|e_{+}, n\right\rangle$ et $\left|e_{-}, n\right\rangle$ (cf. fig. 3).

Supposons cette condition remplie et effectuons le produit des deux rectangles de $\left(\mathrm{D}_{14}\right)$ compris entre l'état résonnant $|a\rangle$ ou $|b\rangle$ et l'état final de la diffusion. L'ordre de ce produit est égal à :

$$
\left|n^{\prime}-n+q\right|+\left|n^{\prime}-n+q-2 p\right|
$$

et se trouve lui aussi minimal et égal à $2 p$ à la condition $n-q \leqslant n^{\prime} \leqslant n-q+2 p$. Autrement dit, il existe $(2 p+1)^{2}$ couples de diagrammes interférant à l'ordre minimum $2 p \times 2=4 p$ dans le champ $\omega_{0}=2 p \omega$. Tous ces couples possèdent deux dénominateurs d'énergie résonnants; ils sont donc du même ordre de grandeur et il faut sommer leurs contributions pour obtenir le signal. Dans le cas de la résonance $\omega_{0}=2 \omega$ par exemple, il faut tenir compte de 9 termes d'interférence. Nous n'en avons envisagé qu'un dans notre étude qualitative (diagrammes $\mathrm{D}_{6} \alpha, \beta$ ). Nous avons représenté un autre couple possible dans

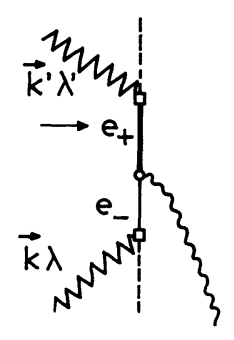

$\alpha)$

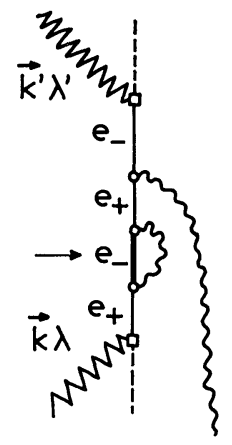

ß)
Diagramme $D_{15}$.
$\left(D_{15} \alpha, \beta\right)$. Les deux niveaux résonnants sont les niveaux $\left|e_{+}, n-1\right\rangle$ et $\left|e_{-}, n+1\right\rangle$ correspondant à la valeur $q=1$. Le nombre de photons se trouve diminué d'une unité dans l'état final : $n^{\prime}=n-1$. Il serait facile de déterminer les sept autres termes d'interférence contribuant à la résonance. Le calcul explicite des signaux va cependant nous montrer que tous ces termes ont la même forme et ne diffèrent les uns des autres que par des coefficients numériques d'intensité. Toutes les considérations qualitatives du $\S$ I établies à partir de l'un d'entre eux restent donc valables.

b) Calcul des signaux de résonance. Interprétation physique. - L'amplitude de transition :

$$
|f n \mathbf{k} \lambda\rangle \rightarrow\left|f n^{\prime} \mathbf{k}^{\prime} \lambda^{\prime}\right\rangle
$$

associée au diagramme passant par le niveau $|a\rangle$ est donnée par la formule (II.B.15). On obtient pour le niveau $|b\rangle$ une expression analogue en remplaçant $a$ par $b$ dans cette formule. Pour calculer la partie du signal qui correspond à l'interférence de ces deux amplitudes, il faut déterminer la partie réelle de leur produit et intégrer, au moyen des résidus, sur l'énergie conformément à (II.G.4). On obtient alors le signal partiel $\left(^{8}\right)$ :

$$
\begin{aligned}
& \mathscr{R} \mathrm{e} \mathscr{S}_{n^{\prime} q}=\text { Cte } \mathscr{R} \mathrm{e} \frac{1}{\Gamma+i\left[E_{a}-E_{b}+\overline{R_{a a}}-\overline{R_{b b}}\right]} \\
& \times\left\langle f n^{\prime} \mathbf{k}^{\prime} \lambda^{\prime}\left|\mathscr{H}_{\mathrm{I}} \bar{F}^{\prime}\right| e_{+}, n-q\right\rangle\left\langle e_{+}, n-q\left|\bar{F} \mathscr{H}_{\mathrm{I}}\right| f n \mathbf{k} \boldsymbol{\lambda}\right\rangle \\
& \times\left\langle f n \mathbf{k} \lambda\left|\mathscr{H}_{\mathrm{I}} \bar{F}^{\prime}\right| e_{-}, n-q+2 p\right\rangle \\
& \times\left\langle e_{-}, n-q+2 p\left|\bar{F} \mathscr{H}_{\mathrm{I}}\right| f n^{\prime} \mathbf{k}^{\prime} \lambda^{\prime}\right\rangle
\end{aligned}
$$

dans lequel les opérateurs $\bar{F}, \bar{F}^{\prime}$ et $\bar{R}$ sont relatifs aux niveaux résonnants $|a\rangle$ et $|b\rangle$. Si $n^{\prime}$ et $q$ sont choisis conformément aux conditions du paragraphe précédent, le produit des éléments de matrice au numérateur de $\mathscr{S}_{n^{\prime} q}$ est d'ordre $4 p$ en $V$. Le signal total s'écrit finalement :

$$
\mathscr{S}=\sum_{\substack{0 \leqslant q \leqslant 2 p \\ n-q \leqslant n^{\prime} \leqslant n-q+2 p}} \mathscr{R} \mathrm{e} \mathscr{S}_{n^{\prime} q} .
$$

Tous les dénominateurs d'énergie des $\mathscr{S}_{n^{\prime} q}$ sont identiques car $E_{a}-E_{b}$ est toujours égal à $\omega_{0}-2 p \omega$ et les déplacements $\overline{R_{a a}}$ et $\overline{R_{b b}}$ varient très peu lorsqu'on passe d'un croisement à un autre (cf. appendice 3 ).

Les signaux $\mathscr{S}_{n^{\prime} q}$ ne diffèrent finalement les uns des autres que par un facteur complexe d'intensité $\beta_{n^{\prime} q}$ dépendant des éléments de matrice $B_{+}, B_{-}, B_{+}^{\prime}, B_{-}^{\prime}$

${ }^{(8)}$ Pour établir (II.C.8), on se sert de la relation $\left(\bar{F}^{\prime}\right)^{+}=\bar{F}$ qui se déduit aisément de (II.B.6) et (II.B.8) et du fait que dans $\bar{F}$ et $\bar{F}^{\prime}$ la variable $z$ est prise pour la valeur réelle $\bar{E}$. 
de $\mathscr{H}_{\mathrm{I}}$, donc des polarisations optiques d'excitation et de détection. En posant :

$$
\beta=\sum_{n^{\prime} q} \beta_{n^{\prime} q}
$$

on peut mettre finalement le signal sous la forme générale :

$$
\mathscr{S}=\left(\gamma H_{1}\right)^{4 p} \mathscr{R} \mathrm{e} \frac{\beta}{\Gamma+i\left[\omega_{0}-2 p \omega+\delta_{2 p}\left(\gamma H_{1}\right)^{2}\right]} .
$$

Les nouvelles résonances $\omega_{0}=2 p \omega$ ont ainsi une intensité proportionnelle à $H_{1}^{4 p}$ (pour les valeurs suffisamment faibles de $H_{1}$ ). Elles se présentent sous la forme d'une somme de courbes d'absorption et de dispersion de Lorentz dont les contributions dépendent des polarisations lumineuses d'excitation et de détection. Leur largeur $\Gamma$ est indépendante du niveau de la radiofréquence et ne dépend finalement que de la largeur naturelle du niveau atomique.

Enfin, la résonance subit un déplacement radiatif en $H_{1}^{2}$ vers les faibles valeurs du champ égal à $-\delta_{2 p}\left(\gamma H_{1}\right)^{2}$. Nous donnons en appendice 3 une expression explicite du coefficient $\delta_{2 p}$.

L'interprétation physique des propriétés de ces résonances est très claire : les déplacements radiatifs sont, comme dans le cas des transitions à plusieurs quanta, dus aux transitions virtuelles qui « renormalisent » les propagateurs des états résonnants. Ceux-ci n'étant pas couplés entre eux, il n'y a pas de possibilité de transition réelle de l'un à l'autre, d'où l'absence d'élargissement radiatif des résonances.
Nous avons observé ces résonances et vérifié quantitativement les prévisions théoriques les concernant. Le détail de la description des expériences a été donné par ailleurs [9]. Indiquons simplement ici que dans nos expériences les photons optiques sont diffusés non par un état atomique excité, mais par un état fondamental paramagnétique $\left({ }^{199} \mathrm{Hg}\right.$ spin nucléaire $\left.J=1 / 2\right)$ dont ils effectuent le pompage optique. Toute la théorie précédente se transpose aisément à ce cas : au lieu de considérer les atomes dans l'état excité, il suffit de raisonner de façon parallèle sur les « trous » laissés par l'excitation optique dans l'état fondamental.

4. Influence de la polarisation de la radiofréquence. Dans ce qui précède, nous n'avons envisagé, en général, que le cas d'une polarisation $\sigma$ du champ de radiofréquence, car on peut, sur cet exemple simple, mettre clairement en évidence les deux types de résonance possibles. Il est évident que tous les résultats obtenus se généralisent aisément au cas d'une polarisation quelconque de la radiofréquence : les transitions réelles à un ou plusieurs quanta ne se manifestent que lorsque la polarisation de la radiofréquence permet de coupler entre eux les niveaux de $\mathscr{H}_{0}$ qui se croisent. Lorsqu'au contraire les niveaux de $\mathscr{H}_{0}$ qui se croisent ne sont couplés à aucun ordre, des effets d'interférence ${ }^{(9)}$ entre deux amplitudes de diffusion au voisinage du

$\left.{ }^{9}\right)$ Il est à noter que ces effets existent également dans les champs correspondant aux transitions à plusieurs quanta (voir $D_{13} \beta, \gamma, \delta$ ), mais ils sont alors masqués par la contribution $\omega / \Gamma$ ou $\omega^{2} / \Gamma^{2}$ fois plus importante du terme principal de la diffusion $\left(\mathrm{D}_{13} \alpha\right)$.

\begin{tabular}{|c|c|}
\hline \multirow{4}{*}{$\begin{array}{c}\text { Polarisation } \\
\text { DE LA RADIOFRÉQUENGE } \\
- \\
\sigma_{+}\end{array}$} & Transitions \\
\hline & $\begin{array}{l}\text { A UN OU PLUSIEURS QUANTA } \\
\text { (élargissements radiatifs) }\end{array}$ \\
\hline & - \\
\hline & $\begin{array}{l}\qquad \omega_{0}=\omega \\
\text { résonance magnétique ordinaire (pas de } \\
\text { déplacement radiatif) }\end{array}$ \\
\hline$\sigma$ & $\begin{array}{c}\omega_{0}=(2 p+1) \omega[4] \quad(p \gtreqless 0) \\
\left.\text { (déplacements radiatifs en }\left(\gamma H_{1}\right)^{2}\right)\end{array}$ \\
\hline$\pi$ & \\
\hline Quelconque & $\omega_{0}=p \omega[4]$ \\
\hline $\begin{array}{l}\text { (superposition linéaire } \\
\left.\operatorname{de} \sigma_{+}, \sigma_{-}, \pi\right)\end{array}$ & (déplacements radiatifs en $\left(\gamma H_{1}\right)^{2}$ ) \\
\hline
\end{tabular}

TyPe DE RÉSONANGE

Le cas $\omega_{0}=0$ correspond à la modification de l'effet Hanle due à la radiofréquence [18]. Nous l'étudions en détail dans un autre article. 
croisement de niveaux peuvent donner naissance à de nouvelles résonances aux propriétés totalement différentes : nous avons résumé les propriétés des diverses résonances observables sur un spin 1/2 dans le tableau ci-devant en indiquant les références où elles ont été étudiées en détail.

Conclusion. - En conclusion, nous voyons ainsi qu'il est possible d'interpréter physiquement et quantitativement toutes les propriétés des diverses résonances qui apparaissent sur la lumière diffusée par un atome en interaction avec des photons de radiofréquence. Toute la théorie présentée ici repose sur l'expression (II.A.4) : $T=\mathscr{H}_{\mathrm{I}} G(z) \mathscr{H}_{\mathrm{I}}$ de la matrice de transition. Afin d'expliciter tous les processus physiques en termes de photons de radiofréquence, nous avons choisi ici de développer $G(z)$ en puissances de $V$ (formule (II.A.8)), chaque terme du développement correspondant à l'émission ou l'absorption d'un certain nombre de photons de radiofréquence. La resommation de tous ces processus nous a fait apparaître des énergies renormalisées différentes de celles de l'atome libre. Ceci suggère une autre interprétation de la formule (II.A.4) : entre l'absorption du photon optique incident et l'émission du photon optique diffusé (termes $\mathscr{H}_{\mathrm{I}}$ ), $G(z)$ décrit la propagation du système total « atome + photons de radiofréquence » en interaction, d'hamiltonien $\mathscr{H}_{0}+V$, que l'on peut encore appeler atome « habillé » par les photons de radiofréquence et dont les niveaux d'énergie diffèrent de ceux de l'atome « nu ». Nous approfondirons ce point de vue dans un autre article ou à partir des états propres et des valeurs propres de $\mathscr{H}_{0}+V$, nous retrouverons les résultats précédents et mettrons en évidence certaines propriétés nouvelles de l'atome habillé (moment magnétique, spectre d'absorption...).

Les auteurs tiennent à remercier M. F. Laloë pour les fructueuses discussions qu'ils ont eues avec lui au sujet de cet article.

\section{APPENDICE 1}

\section{Expression quantique de l'interaction dipolaire magnétique}

1. Expression de $V$. - Appelons $\mathbf{H}_{\mathbf{1}}(t)$ le champ de radiofréquence classique. L'interaction dipolaire magnétique s'écrit classiquement :

$$
V_{\mathrm{cl}}=-\gamma \mathbf{H}_{\mathbf{1}}(\mathbf{t}) . \mathbf{J} \text {. }
$$

Lorsqu'on quantifie le champ électromagnétique, on obtient pour le potentiel vecteur $\mathbf{A}$ :

$$
\mathbf{A}=\sum_{\mathbf{k}, \lambda} \frac{\alpha}{\sqrt{k}}\left[a_{\mathbf{k} \lambda} \boldsymbol{\epsilon}_{\lambda} \mathrm{e}^{i \mathbf{k r}}+a_{\mathbf{k} \lambda}^{\dagger} \boldsymbol{\epsilon}_{\lambda}^{*} \mathrm{e}^{-i \mathbf{k r}}\right]
$$

$a_{\mathbf{k} \lambda}^{\dagger}$ et $a_{\mathbf{k} \lambda}$ étant les opérateurs de création et d'annihilation d'un photon de vecteur d'onde $\mathbf{k}$ et de polarisation $\boldsymbol{\epsilon}_{\lambda} ; \alpha$ est une constante.

Le champ magnétique $\boldsymbol{H}_{1}$ s'écrit alors :

$$
\begin{aligned}
\mathbf{H}_{\mathbf{1}}=\operatorname{rot} \mathbf{A} & =-i \sum_{\mathbf{k}} \sum_{\lambda} \frac{\alpha}{\sqrt{k}} \\
& {\left[a_{\mathbf{k} \lambda} \boldsymbol{\epsilon}_{\lambda} \wedge \mathbf{k} \mathrm{e}^{i \mathbf{k r}}-a_{\mathbf{k} \lambda}^{\dagger} \boldsymbol{\epsilon}_{\lambda}^{*} \wedge \mathbf{k} \mathrm{e}^{-i \mathbf{k r}}\right] . \quad\left(\mathrm{A}_{\mathbf{1}} \cdot 3\right) }
\end{aligned}
$$

Nous n'envisageons ici que le cas où un seul mode du champ est rempli, celui dont la polarisation $\boldsymbol{\epsilon}_{\lambda} \wedge \mathbf{k}$ est égale à $\boldsymbol{\epsilon}$ et dont le module du vecteur d'onde est $|\mathbf{k}|=\frac{\omega}{c}$. Dans la double somme de l'expression précédente, nous ne conserverons donc que ce mode et nous obtiendrons :

$$
\mathbf{H}_{\mathbf{1}}=\frac{\alpha}{\sqrt{k}}\left[a \boldsymbol{\epsilon} \mathrm{e}^{i \mathbf{k} \rho}+a^{\dagger} \boldsymbol{\epsilon}^{*} \mathrm{e}^{-i \mathbf{k} \rho}\right]
$$

(afin d'éliminer la constante imaginaire $i$, nous avons changé de phase en posant $\mathbf{r}=\mathbf{r}_{0}+\rho$ avec $\left.\mathbf{k} \mathbf{r}_{0}=\frac{\pi}{2}\right)$.

Comme les dimensions atomiques sont beaucoup plus petites que la longueur d'onde du champ de radiofréquence (approximation dipolaire), on peut poser $\mathrm{e}^{i \mathbf{k} \rho}=1$ et :

$$
\mathbf{H}_{\mathbf{1}}=\frac{\alpha}{\sqrt{k}}\left[a \boldsymbol{\epsilon}+a^{\dagger} \boldsymbol{\epsilon}^{*}\right]
$$

d'où la forme entièrement quantique de $V$ donnée par l'expression (I.A.3) du $\S$ I.A :

$$
V=\lambda \mathbf{J}\left[a \boldsymbol{\epsilon}+a^{\dagger} \boldsymbol{\epsilon}^{*}\right]
$$

où $\lambda=-\frac{\gamma \alpha}{\sqrt{k}}$ est une constante de couplage que nous déterminons plus loin.

2. ÉtUde DE $V$ EN FONGTION DE LA POLARISATION $€$ DE LA RADIOFRÉQUENGE. - Nous pouvons maintenant déterminer $V$ pour n'importe quelle polarisation $\boldsymbol{\epsilon}$ de la radiofréquence. Nous allons envisager trois exemples. Appelons $\mathbf{e}_{x}, \mathbf{e}_{y}$ et $\mathbf{e}_{z}$ les vecteurs unitaires dans les directions $\mathrm{Ox}$, Oy et $\mathrm{Oz}$.

a) Champ linéaire $\pi$ parallèle à $H_{\mathbf{0}}$. - La polarisation est $\boldsymbol{\epsilon}=\mathbf{e}_{z}$ et $V$ s'écrit :

$$
V_{\pi}=\lambda J_{z}\left(a+a^{\dagger}\right)
$$

$V$ ne couple l'état $\left|e_{m}, n\right\rangle$ qu'aux états $\left|e_{m}, n+1\right\rangle$ et $\left|e_{m}, n-1\right\rangle$ de même nombre quantique $m$ : en effet, un photon de polarisation rectiligne transportant un moment angulaire nul le long de sa direction 
de polarisation, son absorption ou son émission ne peut changer le moment angulaire du spin le long de $H_{0}$.

b) Champ tournant $\sigma_{+}$perpendiculaire à $H_{0}$. La polarisation est $\boldsymbol{\epsilon}=\frac{\mathbf{e}_{x}+i \mathbf{e}_{y}}{\sqrt{2}}$ et on a :

$$
\begin{gathered}
V_{\sigma_{+}}=\frac{\lambda}{\sqrt{2}}\left[a J_{+}+a^{\dagger} J_{-}\right] \\
\left(J_{ \pm}=J_{x} \pm i J_{y}\right)
\end{gathered}
$$

$V$ couple alors l'état $\left|e_{m}, n\right\rangle$ aux états $\left|e_{m+1}, n-1\right\rangle$ et $\left|e_{m-1}, n+1\right\rangle$ : en effet, un photon de polarisation circulaire droite transporte une unité de moment angulaire dans sa direction de propagation et son absorption (ou son émission) entraîne le gain (ou la perte) d'une unité de moment angulaire pour le spin. On obtiendrait de même pour un champ tournant circulaire gauche :

$$
V_{\sigma-}=\frac{\lambda}{\sqrt{2}}\left[a J_{-}+a^{\dagger} J_{+}\right]
$$

c) Champ linéaire $\sigma$ perpendiculaire à $H_{0}$. - La polarisation est $\boldsymbol{\epsilon}=\mathbf{e}_{x}$ et on a :

$$
\begin{aligned}
V_{\sigma}=\lambda J_{x}\left(a+a^{\dagger}\right)=\frac{\lambda}{2}[ & \left.a J_{+}+a^{\dagger} J_{-}\right] \\
& +\frac{\lambda}{2}\left[a J_{-}+a^{\dagger} J_{+}\right] \quad\left(\mathrm{A}_{1} \cdot 7\right)
\end{aligned}
$$

$V$ est la somme de deux termes correspondant respectivement à des photons de polarisations circulaires droite et gauche. L'état $\left|e_{m}, n\right\rangle$ est couplé aux états $\left|e_{m+1}, n-1\right\rangle$ et $\left|e_{m-1}, n+1\right\rangle$ (par l'intermédiaire de la composante $\left.\sigma_{+}\right)$, mais aussi aux états $\left|e_{m+1}, n+1\right\rangle$ et $\left|e_{m-1}, n-1\right\rangle$ (par l'intermédiaire de la composante $\sigma_{-}$).

De façon plus précise, chaque élément de matrice de $V$ obéissant à la règle de sélection $\Delta n= \pm 1$, $\Delta m= \pm 1$, deux niveaux ne sont couplés entre eux que si les différences de valeur des nombres quantiques $n$ et $m$ qui les caractérisent ont la même parité : dans le cas d'un spin $1 / 2,\left|e_{+}, n\right\rangle$ est connecté aux niveaux $\left|e_{+}, n \pm 2 p\right\rangle$ (via $2 p-1$ états intermédiaires) et aux niveaux $\left|e_{-}, n \pm(2 p+1)\right\rangle$ (via $2 p$ états intermédiaires).

3. Détermination De la Gonstante de Gouplage $\lambda$. - La constante de couplage $\lambda$ se détermine en comparant la valeur moyenne de l'opérateur champ magnétique à celle du champ classique qu'il représente.

Supposons que le champ soit défini par un état $|N\rangle$ contenant un nombre défini très grand de photons.
Ce champ est incohérent et dans le cas d'une polarisation linéaire peut s'écrire :

$$
H_{1}(t)=H_{1} \cos (\omega t+\varphi)
$$

$\varphi$ étant une fonction aléatoire à répartition uniforme de 0 à $2 \pi$. La valeur moyenne de $\gamma H_{1}$ pour un tel champ est nulle, mais sa valeur quadratique moyenne s'écrit classiquement :

$$
\sqrt{\overline{\gamma H_{1 \mathrm{c} 1}^{2}}}=\gamma H_{1} \sqrt{\overline{\cos ^{2}(\omega t+\varphi)}}=\frac{\gamma H_{1}}{\sqrt{2}}
$$

et quantiquement :

$$
\begin{aligned}
& \sqrt{\overline{\gamma H_{1 \mathrm{q}}^{2}}=\lambda \sqrt{\left\langle N\left|\left(a \boldsymbol{\epsilon}+a^{\dagger} \boldsymbol{\epsilon}^{*}\right)^{2}\right| N\right\rangle}} \\
&=\lambda \sqrt{2 N+1} \simeq \lambda \sqrt{2 N}
\end{aligned}
$$

En comparant ces deux valeurs, on obtient l'expression (I.A.4) du $\oint$ I.A :

$$
\lambda=\gamma H_{1} / 2 \sqrt{N} .
$$

Il est à noter que si on avait envisagé un champ cohérent constitué par une superposition cohérente d'états $|n\rangle$, on aurait trouvé [19] la même valeur pour $\lambda$ (en appelant $N$ le nombre moyen de photons contenu dans le champ).

\section{APPENDICE 2}

\section{Calcul algébrique de $T$}

Nous reprenons les notations du $\S$ II.A et nous écrirons $G(z)$ sous la forme :

$$
G=P G P+P G Q+Q G P+Q G Q . \quad\left(\mathrm{A}_{2} \cdot 1\right)
$$

Les trois premiers termes de cette somme ont été calculés par L. Mower dans la référence [20]. Nous reprenons rapidement sa démonstration. A partir de l'identité :

$$
\left(z-\mathscr{H}_{0}-V\right) G=1
$$

on obtient, en multipliant à droite par $P$, puis à gauche respectivement par $P$ et par $Q$ :

$$
\begin{aligned}
& P\left(z-\mathscr{H}_{0}-V\right) P(P G P)-P V Q(Q G P)=P \quad\left(\mathrm{~A}_{2} .3\right) \\
& -Q V P(P G P)+Q\left(z-\mathscr{H}_{0}-V\right) Q(Q G P)=0
\end{aligned}
$$

(nous nous sommes servis des relations $Q^{2}=Q$, $P^{2}=P, P+Q=1$ et $\left.Q \mathscr{H}_{0} P=0\right)$. 
De $\left(A_{2} \cdot 4\right)$, on tire :

$$
Q G P=\frac{Q}{z-\mathscr{H}_{0}-Q V Q} Q V P(P G P) . \quad\left(\mathrm{A}_{2} .5\right)
$$

On obtiendrait de même :

$$
P G Q=(P G P) P V Q \frac{Q}{z-\mathscr{H}_{0}-Q V Q}
$$

Puis, en remplaçant dans $\left(\mathrm{A}_{2} \cdot 3\right)$ QGP par son expression $\left(\mathrm{A}_{2} .5\right)$ :

$$
\begin{gathered}
{\left[z-\mathscr{H}_{0}-P V P-P V Q \frac{Q}{z-\mathscr{H}_{0}-Q V Q} Q V P\right]} \\
P G P=P . \quad\left(\mathrm{A}_{2} .7\right)
\end{gathered}
$$

Soit encore :

$$
P G P=\frac{P}{z-\mathscr{H}_{0}-P R P}
$$

à condition de poser :

$$
R=V+V \frac{Q}{z-\mathscr{H}_{0}-Q V Q} V .
$$

Nous retrouvons ainsi les expressions (II.B.10) et (II.B.9) établies diagrammatiquement au § II.B.

Afin d'obtenir $Q G Q$, multiplions maintenant $\left(\mathrm{A}_{2} \cdot 2\right)$ à droite et à gauche par $Q$. On obtient :

$Q\left(z-\mathscr{H}_{0}-V\right)(P G Q+Q G Q)=Q \quad\left(\mathrm{~A}_{2} \cdot 10\right)$

soit :

$$
\left[z-\mathscr{H}_{0}-Q V Q\right] Q G Q=Q+Q V P(P G Q) .
$$

En remplaçant dans $\left(\mathrm{A}_{2} .11\right) P G Q$ par son expression $\left(A_{2} \cdot 6\right)$, on obtient :

$$
\begin{aligned}
& Q G Q=\frac{Q}{z-\mathscr{H}_{0}-Q V Q} \\
& +\frac{Q}{z-\mathscr{H}_{0}-Q V Q} V P G P V \frac{Q}{z-\mathscr{H}_{0}-Q V Q} .
\end{aligned}
$$

En regroupant les expressions $\left(\mathrm{A}_{2} \cdot 5\right),\left(\mathrm{A}_{2} \cdot 6\right)$, $\left(A_{2} .8\right)$ et $\left(A_{2} .12\right)$ dans $\left(A_{2} \cdot 1\right)$, on obtient finalement :

$$
\begin{aligned}
& G=\frac{Q}{z-\mathscr{H}_{0}-Q V Q}+\left(P+\frac{Q}{z-\mathscr{H}_{0}-Q V Q} V P\right) \\
& \frac{P}{z-\mathscr{H}_{0}-P R P}\left(P V \frac{Q}{z-\mathscr{H}_{0}-Q V Q}+P\right) \quad\left(\mathrm{A}_{2} .13\right)
\end{aligned}
$$

ce qui donne, en introduisant les opérateurs $K, F$ et $F^{\prime}$ définis au $\S$ II.B :

$$
T=\mathscr{H}_{\mathrm{I}} G \mathscr{H}_{\mathrm{I}}=\mathscr{H}_{\mathrm{I}} K \mathscr{H}_{\mathrm{I}}+\mathscr{H}_{\mathrm{I}} F \frac{P}{z-\mathscr{H}_{0}-P R P} F^{\prime} \mathscr{H}_{\mathrm{I}}
$$

Nous retrouvons ainsi algébriquement l'expression (II.B.11) de la matrice $T$, établie diagrammatiquement au $\S$ II. B.

\section{APPENDICE 3}

\section{Calcul explicite des déplacements radiatifs des deux types de résonance}

Dans le cas d'un spin $J=1 / 2$ et d'un champ de radiofréquence en polarisation $\sigma$, le déplacement de la raie de résonance magnétique $\omega_{0}=p \omega$ (transition à plusieurs quanta si $p$ est impair, nouvelles résonances si $p$ est pair) est donné par le calcul de la quantité du second ordre $\overline{R_{a a}}(\bar{E})-\overline{R_{b b}}(\bar{E})$ avec :

$$
\begin{aligned}
|a\rangle & =\left|e_{-}, n\right\rangle, \quad|b\rangle=\left|e_{+}, n-p\right\rangle \\
\bar{E} & =-\frac{\omega_{0}}{2}+n \omega=\left(n-\frac{p}{2}\right) \omega .
\end{aligned}
$$

Nous avons :

$$
\begin{aligned}
\overline{R_{a a}}(\bar{E}) & =\frac{\left\langle e_{-}, n|V| e_{+}, n-1\right\rangle\left\langle e_{+}, n-1|V| e_{-}, n\right\rangle}{\left(n-\frac{p}{2}\right) \omega-\frac{\omega_{0}}{2}-(n-1) \omega} \\
+ & \frac{\left\langle e_{-}, n|V| e_{+}, n+1\right\rangle\left\langle e_{+}, n+1|V| e_{-}, n\right\rangle}{\left(n-\frac{p}{2}\right) \omega-\frac{\omega_{0}}{2}-(n+1) \omega} \\
& =-\frac{\lambda^{2}}{4}\left[\frac{n}{(p-1) \omega}+\frac{n+1}{(p+1) \omega}\right] \quad\left(\mathrm{A}_{3} .1\right)
\end{aligned}
$$

et de même :

$\overline{R_{b b}}(\bar{E})=\frac{\lambda^{2}}{4}\left[\frac{n-p+1}{(p-1) \omega}+\frac{n-p}{(p+1) \omega}\right]$

d'où :

$\overline{R_{a a}}(\bar{E})-\overline{R_{b b}}(\bar{E})=\frac{\lambda^{2}}{2}(2 n-p+1) \frac{p}{\left(p^{2}-1\right) \omega}$.

Notons au passage sur la formule $\left(\mathrm{A}_{3} \cdot 3\right)$ que $\overline{R_{a a}}-\overline{R_{b b}}$ pour une résonance $\omega_{0}=p \omega$ donnée dépend en toute rigueur du croisement $I_{n}^{p}$ des niveaux envisagés. Cependant, lorsqu'on passe de $I_{n}^{p}$ à $I_{n+1}^{p}$, la variation relative de $\overline{R_{a a}}-\overline{R_{b b}}$ est égale à $1 / n$, ce 
qui est totalement négligeable étant donné le très grand nombre de photons contenus dans le champ de radiofréquence. Il est donc parfaitement justifié de négliger, comme on le fait au § II.C.3, la variation de la quantité $\overline{R_{a a}}-\overline{R_{b b}}$ pour les différents croisements de niveaux alignés sur une même verticale $\omega_{0}=p \omega$.

Le nombre de photons $n$ est très grand devant $p$, et on peut écrire :

$$
2 n-p+1 \sim 2 n .
$$

On obtient finalement le déplacement :

$$
\begin{aligned}
\overline{R_{a a}}-\overline{R_{b b}} & =-\lambda^{2} n \frac{p}{\left(p^{2}-1\right) \omega} \\
& =-\frac{\left(\gamma H_{1}\right)^{2}}{4} \frac{p}{\left(p^{2}-1\right) \omega}
\end{aligned}
$$

(en remplaçant $\lambda$ par son expression (I.A.4)).

D'où le coefficient :

$$
\delta_{p}=p / 4\left(p^{2}-1\right) \omega .
$$

Le calcul précédent ne s'applique qu'au cas $p \neq 1$. Pour déterminer le déplacement de la résonance $\omega_{0}=\omega$, il faut reprendre le calcul de $\overline{R_{a a}}$ et $\overline{R_{b b}}$ avec $|a\rangle=\left|e_{-}, n\right\rangle$ et $|b\rangle=\left|e_{+}, n-1\right\rangle$. La présence du projecteur $Q$ dans le développement de $R$ fait que $\overline{R_{a a}}$ et $\overline{R_{b b}}$ n'ont plus qu'un seul terme :

$$
\begin{array}{r}
\overline{R_{a a}}=\frac{\left\langle e_{-}, n|V| e_{+}, n+1\right\rangle\left\langle e_{+}, n+1|V| e_{-}, n\right\rangle}{\left(n-\frac{1}{2}\right) \omega-\frac{\omega_{0}}{2}-(n+1) \omega} \\
=-\frac{\lambda^{2}}{4} \frac{n+1}{2 \omega} .
\end{array}
$$

On trouve de même :

$$
\overline{R_{b b}}=\frac{\lambda^{2}}{4} \frac{n-1}{2 \omega}
$$

et :

$$
\overline{R_{a a}}-\overline{R_{b b}}=-\lambda^{2} n / 4 \omega=-\delta_{1}\left(\gamma H_{1}\right)^{2}
$$

avec :

$$
\delta_{1}=1 / 16 \omega
$$

\section{APPENDICE 4}

\section{Etude quantique de la modulation des signaux résonnants}

Jusqu'à présent, nous avons considéré que le champ de radiofréquence était un champ incohérent représenté par un état $|n\rangle$. En fait, la phase des champs de radiofréquence réalisés habituellement est très bien définie et le modèle précédent ne peut rendre compte des phénomènes de modulation des signaux résonnants. Afin de les interpréter, nous devons considérer que le champ de radiofréquence est un état cohérent de Glauber [21] :

$$
|\psi(t)\rangle=\sum_{n} C_{n} \mathrm{e}^{-i n \omega t}|n\rangle
$$

le nombre moyen $N$ de photons dans le champ s'écrivant :

$$
N=\sum_{n}\left|C_{n}\right|^{2} n
$$

et la probabilité $\left|C_{n}\right|^{2}$ de trouver $n$ photons dans le champ variant suivant une loi de Poisson centrée en $N$ et de largeur $\sqrt{N}$. Étant donné le nombre extrêmement élevé de photons présents, les coefficients $C_{n}$ que l'on peut toujours supposer réels restent donc sensiblement constants sur un très large intervalle autour de $N$.

Connaissant l'état initial $|f \psi \mathbf{k} \boldsymbol{\lambda}\rangle$ du système, nous allons déterminer l'amplitude de probabilité pour que l'état final de la diffusion soit un état $\left|f n^{\prime} \mathbf{k}^{\prime} \lambda^{\prime}\right\rangle$.

Pour cela, nous allons passer en représentation d'interaction par rapport à l'hamiltonien $\mathscr{H}_{0}+\mathscr{H}_{\text {opt }}$ et écrire que l'amplitude de diffusion cherchée est égale à l'élément de la matrice de collision $S$ entre l'état initial et l'état final de la diffusion [13]; l'état initial $|i\rangle$ de la diffusion s'écrit dans cette représentation :

$$
|i\rangle=\sum_{n_{1}} C_{n_{1}}\left|f n_{\mathbf{1}} \mathbf{k} \boldsymbol{\lambda}\right\rangle
$$

et l'élément de matrice de $S$ :

$$
\begin{aligned}
\left\langle f n^{\prime} \mathbf{k}^{\prime} \lambda^{\prime}|S| i\right\rangle= & \sum_{n_{1}} C_{n_{1}}\left\langle f n^{\prime} \mathbf{k}^{\prime} \lambda^{\prime}|S| f n_{\mathbf{1}} \mathbf{k} \lambda\right\rangle \\
= & -2 i \pi \sum_{n_{1}} C_{n_{1}}\left\langle f n^{\prime} \mathbf{k}^{\prime} \lambda^{\prime}|T| f n_{\mathbf{1}} \mathbf{k} \boldsymbol{\lambda}\right\rangle \\
& \delta\left[E_{k}-E_{k^{\prime}}+\left(n_{\mathbf{1}}-n^{\prime}\right) \omega\right] \quad\left(\mathrm{A}_{\mathbf{4}} .1\right)
\end{aligned}
$$

(nous supposons toujours $\mathbf{k}^{\prime} \neq \mathbf{k}$ ).

L'état final $|d\rangle$ de la diffusion s'écrit alors, si nous imposons la polarisation $\mathbf{e}_{\lambda^{\prime}}$ et la direction de la lumière diffusée :

$$
|d\rangle=\sum_{n^{\prime} k^{\prime}}\left|f n^{\prime} \mathbf{k}^{\prime} \lambda^{\prime}\right\rangle\left\langle f n^{\prime} \mathbf{k}^{\prime} \lambda^{\prime}|S| i\right\rangle
$$

ou encore, sous forme de matrice densité :

$$
\begin{aligned}
|d\rangle\left\langle d\left|=\sum_{\substack{n^{\prime} n^{\prime \prime} \\
k^{\prime} k^{\prime \prime}}}\right| f n^{\prime} \mathbf{k}^{\prime} \lambda^{\prime}\right\rangle\left\langle f n^{\prime} \mathbf{k}^{\prime} \lambda^{\prime}|S| i\right\rangle & \left\langle i|S| f n^{\prime \prime} \mathbf{k}^{\prime \prime} \lambda^{\prime}\right\rangle\left\langle f n^{\prime \prime} \mathbf{k}^{\prime \prime} \lambda^{\prime}\right| . \quad\left(\mathbf{A}_{\mathbf{4}} \cdot 2\right)
\end{aligned}
$$

La matrice densité $\rho$ du champ optique s'obtiendra en prenant la trace de $|d\rangle\langle d|$ par rapport au champ 
de radiofréquence et aux variables atomiques : on obtient finalement, en faisant $n^{\prime}=n^{\prime \prime}$ dans $\left(\mathrm{A}_{4} \cdot 2\right)$ et en sommant sur $n^{\prime}$ :

$$
\begin{aligned}
& \rho=\operatorname{Tr}_{\text {at, rf }}|d\rangle\langle d| \\
& =\sum_{\substack{n^{\prime} \\
k^{\prime} k^{\prime \prime}}}\left|\mathbf{k}^{\prime} \lambda^{\prime}\right\rangle\left\langle f n^{\prime} \mathbf{k}^{\prime} \lambda^{\prime}|S| i\right\rangle\left\langle i|S| f n^{\prime} \mathbf{k}^{\prime \prime} \lambda^{\prime}\right\rangle\left\langle\mathbf{k}^{\prime \prime} \lambda^{\prime}\right|
\end{aligned}
$$

ou encore en explicitant dans $\left(A_{4} \cdot 3\right)$ les éléments de matrice de $S$ donnés par $\left(\mathrm{A}_{\mathbf{4}} \cdot 1\right)$ : $\rho=4 \pi^{2} \sum_{n^{\prime}} \sum_{k^{\prime} k^{\prime \prime}} \sum_{n_{1} n_{2}}\left|k^{\prime} \lambda^{\prime}\right\rangle\left\langle f n^{\prime} \mathbf{k}^{\prime} \lambda^{\prime}|T| f n_{1} \mathbf{k} \lambda\right\rangle C_{n_{1}} C_{n_{2}}^{*}$
$\left\langle f n_{2} \mathbf{k} \lambda|T| f n^{\prime} \mathbf{k}^{\prime \prime} \lambda^{\prime}\right\rangle\left\langle\mathbf{k}^{\prime \prime} \lambda^{\prime}\right|$

$\delta\left[E_{k}-E_{k^{\prime}}+\left(n_{1}-n^{\prime}\right) \omega\right] \delta\left[E_{k}-E_{k^{\prime \prime}}+\left(n_{2}-n^{\prime}\right) \omega\right]$.

Soit encore, en remaniant les fonctions $\delta$ :

$$
\rho=\sum_{k^{\prime} k^{\prime \prime}}\left|\mathbf{k}^{\prime} \lambda^{\prime}\right\rangle \rho_{k^{\prime}} k^{\prime \prime}\left\langle\mathbf{k}^{\prime \prime} \lambda^{\prime}\right|
$$

avec :

$$
\begin{aligned}
& \rho_{k^{\prime} k^{\prime \prime}}=\text { Cte } \sum_{n^{\prime}} \sum_{n_{1} n_{2}}\left\langle f n^{\prime} \mathbf{k}^{\prime} \lambda^{\prime}|T| f n_{\mathbf{1}} \mathbf{k} \lambda\right\rangle C_{n_{1}} C_{n_{2}}^{*} \\
& \left\langle f n_{\mathbf{2}} \mathbf{k} \lambda|T| f n^{\prime} \mathbf{k}^{\prime \prime} \lambda^{\prime}\right\rangle \delta\left[E_{k^{\prime}}-E_{k^{\prime \prime}}-\left(n_{\mathbf{1}}-n_{\mathbf{2}}\right) \omega\right] \\
& \quad \delta\left[E_{k}-E_{k^{\prime}}+\left(n_{\mathbf{1}}-n^{\prime}\right) \omega\right] . \quad\left(\mathrm{A}_{\mathbf{4}} \cdot \mathbf{5}\right)
\end{aligned}
$$

La matrice densité du rayonnement présente ainsi des « cohérences » entre états dont la différence d'énergie correspond aux divers harmoniques $\left(n_{1}-n_{2}\right) \omega$ de la fréquence du champ de radiofréquence. En d'autres termes, l'onde optique diffusée par le système dans une direction donnée est constituée par une superposition cohérente d'ondes monochromatiques de même direction de propagation dont les fréquences diffèrent de multiples de $\omega$ et qui sont donc susceptibles de battre entre elles.

Afin de calculer le signal optique $\mathscr{S}$ correspondant, nous supposerons que nous avons un détecteur à bande passante large situé au point $r$ qui est sensible à la valeur moyenne de la fonction de corrélation [22] :

$$
\mathscr{G}(r t, r t)=E_{+}(r, t) E_{-}(r, t)
$$

$E_{-}$et $E_{+}$étant les composantes à fréquence négative et positive de l'opérateur champ électrique quantifié.
On obtient alors aisément, à partir de $\left(\mathrm{A}_{4} .5\right)$ et après pondération sur la distribution spectrale du rayonnement incident :

$$
\begin{aligned}
\mathscr{S}=\text { Cte } \sum_{n^{\prime}} \sum_{n_{1} n_{2}} \iiint U\left(E_{k}\right) \mathrm{d} E_{k} \mathrm{~d} E_{k^{\prime}} \mathrm{d} E_{k^{\prime \prime}} \\
\begin{array}{r}
\delta\left[E_{k^{\prime}}-E_{k^{\prime \prime}}-\left(n_{1}-n_{\mathbf{2}}\right) \omega\right] \delta\left[E_{k}-E_{k^{\prime}}+\left(n_{\mathbf{1}}-n^{\prime}\right) \omega\right] \\
\left\langle f n^{\prime} \mathbf{k}^{\prime} \lambda^{\prime}|T| f n_{\mathbf{1}} \mathbf{k} \lambda>C_{n_{1}} C_{n_{2}}^{*}\right. \\
\left\langle f n_{\mathbf{2}} \mathbf{k} \boldsymbol{\lambda}|T| f n^{\prime} \mathbf{k}^{\prime \prime} \lambda^{\prime}>\mathrm{e}^{-i\left(n_{1}-n_{2}\right) \omega t .} \quad\left(\mathrm{A}_{\mathbf{4}} \cdot 6\right)\right.
\end{array}
\end{aligned}
$$

Remarquons que l'expression précédente s'applique au cas d'un champ $|\psi\rangle=|n\rangle$ incohérent à condition d'y faire $n_{1}=n_{2}=n$ : les modulations disparaissent, la première fonction $\delta$ impose $k^{\prime}=k^{\prime \prime}$ et nous sommes ramenés exactement à l'expression (II.C.2) $\left.{ }^{10}\right)$.

Nous voyons ainsi que les modulations apparaissant sur les signaux optiques sont dues à l'interférence entre les deux amplitudes $\left\langle f n^{\prime} \mathbf{k}^{\prime} \lambda^{\prime}|T| f n_{\mathbf{1}} \mathbf{k} \lambda\right\rangle$ et $\left\langle f n^{\prime} \mathbf{k}^{\prime \prime} \lambda^{\prime}|T| f n_{2} \mathbf{k} \boldsymbol{\lambda}\right\rangle$. Les deux états initiaux $\left|f n_{1} \mathbf{k} \lambda\right\rangle$ et $\left|f n_{2} \mathbf{k} \boldsymbol{\lambda}\right\rangle$ sont distincts car ils contiennent des nombres de photons de radiofréquence différents. Ils présentent cependant une relation de phase bien définie par suite du caractère cohérent du champ de radiofréquence. Cette relation de phase se retrouve, après la diffusion, dans le champ optique, les deux états finaux $\left|f n^{\prime} \mathbf{k}^{\prime} \lambda\right\rangle$ et $\left|f n^{\prime} \mathbf{k}^{\prime \prime} \lambda^{\prime}\right\rangle$ contenant le même nombre de photons de radiofréquence mais correspondant à des fréquences optiques différentes.

En explicitant les deux amplitudes précédentes et en effectuant sur elles les mêmes resommations que celles du $\S$ II. B, on peut déterminer toutes les propriétés des résonances apparaissant sur les modulations des signaux optiques. Il résulte de façon évidente de ce calcul que les propriétés intrinsèques de ces résonances (position, élargissement, déplacement) sont les mêmes, quelles que soient les modulations sur lesquelles elles sont détectées et identiques à celles des résonances étudiées au § II.C (signal statique). Un tel résultat est beaucoup plus difficile à démontrer lorsqu'on traite classiquement le champ de radiofréquence [23].

(10) A condition de poser $\left|C_{n}\right|^{2}=1$.

\section{BIBLIOGRAPHIE}

[1] Barrat (J. P.) et Cohen-Tannoudji (C.), J. Physique Rad., 1961, 22, 329 et 443.

[2] Cohen-Tannoudji (C.), Thèse, Paris, 1962 ; Ann. Physique, 1962, 7, 423 et 469.

[3] Wallace (R.), Molecular Physics, 1966, 11, 5, 457.

[4] Winter (J. M.), Thèse, Paris, 1958 ; Ann. Physique, $1959,4,745$.
[5] Dodd (J. N.), Serres (G. W.) et Taylor (M. J.), Proc. Roy. Soc., 1963, A 273, 41.

[6] Alexandrov (E. B.), Constantinov (O. B.), PERELI (B. I.) et KHodovoy (B. A.), J.E.T.P. (USSR), 1963, 45, 503.

[7] Favre (C. J.) et Geneux (E.), Phys. Letters, $1964,8,3,190$. 
[8] Polonsky (N.) et Cohen-Tannoudji (C.), C. $R$. Acad. Sci., 1965, 260, 5231.

[9] Cohen-Tannoudji (C.) et Haroche (S.), C. R. Acad. Sci., 1965, 261, 5400.

[10] Pour les représentations diagrammatiques des amplitudes de transition, voir par exemple Wick (G. C.), Rev. Mod. Phys., 1955, 27, 339, ou encore les références [3] ou [15].

[11] Khodovoy (B. A.), J.E.T.P. (USSR), 1964, 46, 331.

[12] Hanle (W.), Z. Phys., 1924, 30, 93.

[13] Messiah (A.), Mécanique quantique, tome II, Dunod, 1964.

[14] Goldberger (M. L.) et WaTson (K. M.), Collision Theory (John Wiley and Sons Inc.).

[15] Cohen-Tannoudji (C.), Compléments de Mécanique quantique (cours de $3^{\mathrm{e}}$ cycle, polycopié, Paris, 1966).
[16] Cohen-Tannoudji (C.), Cargese Lectures in Physics, Gordon and Breach, 1967.

[17] Bi,och (F.) et SiegerT (A.), Phys. Rev., 1940, 57, 522 .

[18] Cohen-Tannoudji (C.) et Haroche (S.), C. R. Acad. Sci., 1966, 262, 268.

[19] Polonsky (N.) et Cohen-Tannoudji (C.), J. Physique, 1965, 26, 409.

[20] Mower (L.), Phys. Rev., 1966, 142, 4, 799.

[21] Glauber (R. J.), Phys. Rev., 1963, 131, 6, 27662788.

[22] GLAUBER (R. J.), Quantum Theory of Radiation (Optique et électronique quantiques, Les Houches, 1964, Gordon and Breach).

[23] Cohen-Tannoudji (C.) et Haroche (S.), C. R. Acad. Sci., 1967, 264, 626.

Haroche (S.), Thèse de $3^{\text {e }}$ cycle, Paris, 1967.

Le Directeur de la Publication : Jules-Jean Chovin. - Imprimerie des Presses Universitaires de France, Vendôme (France) Dépôt légal : 1-1969 